\title{
Network Pharmacology and Molecular Docking Study of the Chinese Miao Medicine Sidaxue in the Treatment of Rheumatoid Arthritis
}

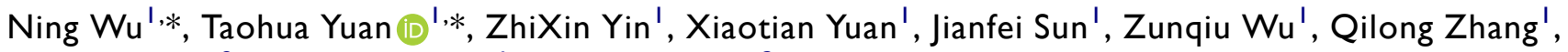 \\ Carl Redshaw ${ }^{2}$, Shenggang Yang ${ }^{\prime}$, Xiaotian Dai $\mathbb{D}^{3}$ \\ 'Guizhou Medical University, Guiyang, Guizhou, People's Republic of China; ${ }^{2}$ Department of Chemistry, University of Hull, Hull, Yorkshire, HU6 7RX, \\ UK; ${ }^{3}$ Department of Mathematics and Statistics, University of Calgary, Calgary, AB, Canada \\ *These authors contributed equally to this work \\ Correspondence: Shenggang Yang, Guizhou Medical University, Guiyang, Guizhou, 550025, People's Republic of China, Tel/Fax +86 I3I58000576, \\ Email yangshenggang2021@|26.com; Xiaotian Dai, Department of Mathematics and Statistics, University of Calgary, Calgary, AB, T2N IN4, Canada, \\ Tel/Fax + 435754 4980, Email xiaotian.dai@uCalgary.ca
}

Purpose: This study aimed to investigate the molecular mechanisms of Compound Sidaxue (SX), a prescription of Chinese Miao medicine, in treating rheumatoid arthritis (RA) using network pharmacology and in vivo experimental approaches.

Methods: Network pharmacology was adopted to detect the active components of four Traditional Chinese herbal medicine (TCM) of $\mathrm{SX}$, and the key targets and signaling pathways in the treatment of RA were predicted, and the key components and targets were screened for molecular docking. The predicted targets and pathways were validated in bovine type II collagen and incomplete Freund's adjuvant emulsifier-induced rat RA model.

Results: In this study, we identified 33 active components from SX, predicted to act on 44 RA-associated targets by network pharmacology. PPI network demonstrated that TNF- $\alpha$, VEGF-A, IL-2, IL-6, AKT, PI3K, STAT1 may serve as the key targets of SX for the treatment of RA. The main functional pathways involving these key targets include PI3K-AKT signaling pathway, TNF signaling pathway, NF-kB signaling pathway. Molecular docking analysis found that the active components $\beta$-amyrin, cajanin, eleutheroside A have high affinity for TNF- $\alpha$, VEGFA, IL-2, AKT, and PI3K, etc. SX can improve joint swelling in Collagen-induced arthritis (CIA) rats, reduce inflammatory cell infiltration and angiogenesis in joint synovial tissue, and down-regulate IL-2, IL-6, TNF- $\alpha$, VEGF,

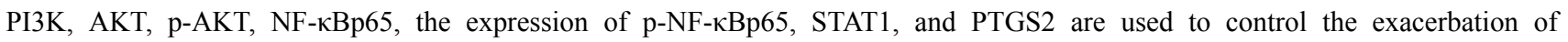
inflammation and alleviate the proliferation of synovial pannus, and at the same time play the role of cartilage protection to achieve the effect of treating RA.

Conclusion: Through a network pharmacology approach and animal study, we predicted and validated the active compounds of SX and their potential targets for RA treatment. The results suggest that SX can markedly alleviate CIA rat by modulating the VEGF/ $\mathrm{PI} 3 \mathrm{~K} / \mathrm{AKT}$ signaling pathway, TNF- $\alpha$ signaling pathway, IL/NF- $\mathrm{BB}$ signaling pathway.

Keywords: network pharmacology, molecular docking, rheumatoid arthritis, Sidaxue, VEGF/PI3K/AKT signaling pathway, TNF- $\alpha$

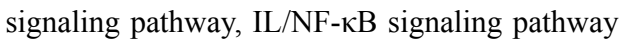

\section{Introduction}

Rheumatoid arthritis (RA) is a common type of chronic inflammatory autoimmune disease that primarily affects joints, but in severe cases, also affects internal organs, such as the lung, heart, and kidney. RA is clinically characterized by a sudden onset of chronic and progressive joint swelling, pain, and joint deformities, which gradually cause joint dysfunction and eventually disability. ${ }^{1-3}$ RA, as a refractory disease, has the highest disability rate among all joint diseases, affecting between $0.5 \%$ and $1 \%$ of the global population, and is most common amongst the elderly. Further analysis reveals that the incidence for women is 2 to 3 times that of men, and is one of the main reasons for human loss of 
labor and disability. ${ }^{1-3}$ At present, there is no clinically effective drug for the treatment of RA, and commonly used drugs have certain unwanted side effects. ${ }^{4,5}$ For example, nonsteroidal anti-inflammatory drugs (NSAIDs), which are drugs that are commonly used to improve the symptoms of arthritis, have analgesic and anti-inflammatory effects, however they cannot control the development of the disease and have obvious gastrointestinal reactions. Moreover, they can increase the incidence of cardiovascular disease. Disease modifying anti-rheumatic drugs (DMARDs) have the effect of improving and delaying the progress of the disease. However, the onset is slow, and can take 1-6 months to be effective, and it is easy to cause liver, gastrointestinal, bone marrow problems, whilst the function of the kidneys and other organs can be impaired. Glucocorticoid (GC) has a great anti-inflammatory effect and can quickly relieve joint swelling and pain as well as systemic symptoms. However, due to its extensive side effects, it has not been used for some time. Biological agents include tumor necrosis factor- $\alpha$ (TNF- $\alpha$ ) antagonists, interleukin-1 (IL-1) antagonists, interleukin-6 (IL-6) antagonists, targeted small molecule drugs, etc., which have significant curative effects, but increase the prevalence of infections and tumor diseases. ${ }^{5-8}$ Due to the obvious side effects of these drugs, their effect on the treatment of RA is limited, and some drugs have a single mechanism of action, which cannot fundamentally solve the development and onset of RA. Therefore, it is particularly necessary to develop new drugs which exhibit limited side effects and significant curative effects. ${ }^{4-6}$ It is noteworthy that Chinese herbal medicine has the characteristics of multi-component, multipathway, and a multi-target action, with fewer side effects, and can play an important role in the prevention and treatment of RA diseases. ${ }^{9-12}$ GTW is a classic Miao medicine with a clear curative effect in the treatment of RA among clinical drugs, and most studies of Chinese medicine formula use GTW as the positive drug, therefore, this study uses GTW as a positive control group. ${ }^{5}$

The Miao medicine Sidaxue (SX) is a representative anti-rheumatic prescription that has been widely used to treat rheumatic pain and blood stasis by the minority ethnic Miao group in the northern part of Guizhou Province in China. SX is a concoction made of four Miao herbal medicines: ji-xue-teng (JXT) (Spatholobus suberectus Dunn), da-xue-teng (DXT) (Sargentodoxa cuneata (Oliv.) Rehd. et Wils.), fei-long-zhang-xue-geng-pi (FLZXGP) (Toddalia asiatica L. Lam.), and hei-gu-teng (HGT) (Periploca forrestii Schltr). ${ }^{13-15}$ The traditional Chinese medicine JXT is the dried cane of the legume Spatholobus suberectus Dunn. It is mainly distributed in Guangdong, Guangxi, Yunnan and other places. It has the effects of invigorating blood, regulating menstruation and pain, relaxing muscles and collaterals. It is used for irregular menstruation, Blood deficiency, chlorosis, numbness, paralysis, rheumatic arthralgia, etc. ${ }^{16,17}$ Its common prescriptions include Self made ningleg soup, ${ }^{18}$ jixueteng keli, ${ }^{19}$ angelica and caulis decoction, etc., The stem of Spatholobus suberectus Dunn, known as "Da Xue Teng" in Chinese, widely distributed in the subtropical zone of China. The plant is extensively used in traditional medicine for treating arthritis, joint pains, amenorrhea, acute appendicitis, and inflammatory intestinal obstruction, it has been widely used in the treatment of various diseases in China over nine hundred years. In Chinese pharmacopoeia (2020), it possesses abilities of removing toxins (detoxicant) and furuncles, clearing heat, invigorating blood circulation, promoting the flow of channels, dispelling wind, and relieving rheumatic conditions, and is used to treat arthritis and amenorrhea. Its medicinal forms and uses vary from decoctions for oral administration to washing wounds. ${ }^{20,21}$ FLZXGP belonging to Toddalia genus of Rutaceae family, is a folk medicine in China used for hundreds of years, it is produced in various places south of the southern slope of the Qinling Mountains, the whole plant can be used as medicine, especially the root that used to be applied in the folk. The "Quality Standard of Traditional Chinese Medicinal Materials and National Medicinal Materials in Guizhou Province" records that FLZXGP has the effects of dispelling wind and pain, dispelling blood stasis and stopping bleeding, relieving swelling and detoxification, and is mainly used to treat rheumatic arthralgia, stomach pain, and fall and flutter injury. ${ }^{22,23}$ HGT was listed as a classical medicinal plant in "Miao medicine", which is a branch of traditional Chinese medicine (TCM), it is mainly produced in Guizhou, and it is also distributed in Tibet, Yunnan, Guangxi, Qinghai and other places in China. According to the theory of TCM, P. forrestii has the efficacy of relaxing tendons and activating collaterals, and dispelling wind and eliminating dampness. Hence, it was often used for the therapy of rheumatoid arthritis and traumatic injury in clinical practice. ${ }^{24-26}$ Preliminary research by the project team has shown that the toxic effects of SX are small, whilst the lethal dose (lethal dose 50\%, LD50) is $404.18 \mathrm{~g} / \mathrm{kg}$, which is about 802 times that of Tripterygium wilfordii, which is currently the drug of choice for the treatment of RA by traditional Chinese medicine (triptery wilfordii LD50: $504.0 \pm 29.48 \mathrm{mg} / \mathrm{kg}$ ). The efficacy of SX in the treatment of adjuvant arthritis (AA) rats is 
similar to that of positive drug Tripterygium wilfordii, and furthermore SX can alleviate the joint swelling of Freund's adjuvant-induced arthritis AA rats, but the specific mechanism of action is unknown. ${ }^{13-15}$ Traditional Chinese Medicine (TCM) has a long history of viewing an individual or patient as a system with varying status and has over the years accumulated numerous herbal formulae. The holistic philosophy of TCM shares much with the key ideas of emerging both network pharmacology and biology, and meets the requirements of overcoming complex diseases. Network pharmacology is a technique used to systematically evaluate the pharmacological effects of drugs with multiple components and multiple targets by establishing links between targets, drugs, and diseases based on the principles of systems biology, with the latter sharing a similar holistic philosophy to TCM. ${ }^{27-29}$ The components of TCM are complex and diverse, and the composition of the prescription is more complicated. There is an urgent need to establish research strategies and new methods that can reflect the overall characteristics of TCM. ${ }^{27-29}$ In recent years, network pharmacology has integrated the three aspects of the active ingredients of traditional Chinese medicine, related corresponding targets to corresponding diseases, and using them as three different types of nodes to form a "component-target-disease" network. Network analysis methods are used to reveal the effective components and mechanism of action of Chinese medicine in the treatment of related diseases. ${ }^{27-29}$ Therefore, network pharmacology is an important means to analyze the effective components and therapeutic targets of TCM. ${ }^{27-29}$ As a widely used virtual screening method, molecular docking technology can study the interaction between small molecules and receptors, and thereby can predict binding modes and affinities. ${ }^{27}$ At this stage, a number of studies have adopted network pharmacology and molecular docking technology to explore the material basis and mechanism of action of Chinese medicine in treating certain diseases, and have determined the some of the underlying principles and feasibility of these technical methods. ${ }^{30}$

In this study, we have applied network pharmacology and molecular docking through data mining and analysis, to screen and predict potential target molecules and signal pathways for SX active components in RA treatment. Key candidate targets were further verified in animal models of RA. Our results provide molecular insights into the mechanism of Miao medicine prescription SX in the treatment of RA, and will inform further development of anti-RA drugs from SX.

\section{Materials and Methods}

\section{Experimental Animal, Drugs and Reagents}

Sixty specific-pathogen-free Sprague Dawley rats, including 30 females and 30 males, weighing 180-220 g, were purchased from Chongqing Tengxin Biotechnology Co., Ltd (Chongqing, sichuanProvince, China) [License number: $\operatorname{SCXK}(\mathrm{Yu}) 2007.005]$. The four component herbs of SX, ie, JXT, DXT, FLZXGP, and HGT (The ratio is 15:22:15:8), were authenticated by Associate Professor Zhenhua Tian of the Guizhou University of Traditional Chinese Medicine. Tripterygium glycoside tablets (batch number: 20080301) were obtained from Huangshi Feiyun Pharmaceutical Company (Huangshi, Hubei Province, China). Incomplete Freund's adjuvant (lot number: 033K8933) was obtained from Sigma (St. Louis, MO, USA). Rat vascular endothelial growth factor (VEGF-A), TNF- $\alpha$, IL-2, IL-6 enzyme-linked immunoassay (ELISA) kit (MultiScience), PI3 Kinase p110a (C73F8) Rabbit mAb(\#4249), Akt Antibody (\#9272), Phospho-Akt (Ser473) (193H12) Rabbit mAb (\#4058) and NF-kB p65 (D14E12) XP Rabbit mAb (\#8242), Phospho-NF -КB p65 (Ser536) (93H1) Rabbit mAb(\#3033) were purchased from Cell Signaling (Danvers, MA, USA). Anti-rabbit and mouse secondary antibodies were purchased from Bioworld (Beijing, China). The RT-PCR kit was obtained from Baori Biotechnology (Beijing, China).

\section{Equipment}

The following equipment was employed: A SynGene ${ }^{\mathrm{TM}}$ gel imaging system (Synoptics, USA), an ABI StepOnePlus ${ }^{\mathrm{TM}}$ real-time fluorescent quantitative PCR instrument (Thermo, USA), Nanodrop2000 (Thermo Fisher Scientific, Waltham, MA, United States), Microplate reader (Thermo Fisher, USA), Electrophoresis Apparatus(DYY-6D, China), Sorvall ${ }^{\mathrm{TM}}$ ST 8 Small Benchtop Centrifuge(Thermo FisherScientific, USA). 


\section{Identification of SX Active Compounds and Putative Compound Targets}

The active compounds in SX were collected from the TCM Systems Pharmacology Database and Analysis Platform (TCMSP;https://old.tcmsp-e.com/tcmsp.php), ${ }^{31}$ Chemistry Database (http://www.organchem.csdb.cn/scdb/default.htm? nCount $=13303008$ ) ${ }^{32}$ and PubMed (https://pubmed.ncbi.nlm.nih.gov/?term=). ${ }^{32}$ The absorption, distribution, metabolism, and excretion (ADME) profiles of these compounds were obtained via literature searches and used to facilitate the screening of effective ingredients. ${ }^{33}$ The results from the three databases were integrated and duplicate output molecules were removed. To select for active compounds from the four herbal medicines, the raw results were further filtered using two important drug qualitative indicators, ie, oral bioavailability $(\mathrm{OB}$, cut-off $\geq 30 \%)$ and drug-like properties (DL, cutoff $\geq 0.18$ ). Cytoscape Version 3.6.1 software was used to construct the SX-active ingredient map. The corresponding targets of the putative compounds were searched in the TCMSP and the Swiss Target Prediction database (http://www. swisstargetprediction.ch/). ${ }^{35,36}$ To expand the potential number of targets, the molecular similarity match tool, the Simplified Molecular Input Line Entry System (SMILES) in PubChem (https://pubchem.ncbi.nlm.nih.gov/\#query= Nevadensia $^{35}$ was used. These two groups of targets were entered into the UniProt database with the organism defined as "Homo sapiens" and were considered SX potential targets. UniProt (https://www.uniprot.org) was used to find gene names and Uniprot numbers of the putative target genes. ${ }^{35}$

\section{Identification of Rheumatoid Arthritis-Associated Targets}

The key word "Rheumatoid arthritis" was used to search Therapeutic Target Database (TTD) (http://db.idrblab.net/ttd/), ${ }^{34}$ Drugbank (https://www.drugbank.ca/), ${ }^{34}$ and DisGeNET (https://www.disgenet.org/) ${ }^{36}$ databases for RA-associated targets that have been experimentally verified. Intersections of the targets were subjected to deduplication and their UniProt numbers were retrieved. ${ }^{36}$

\section{Network Construction and Pathway Enrichment}

A Venn diagram was drawn from the predicted SX active components and hypothetic RA-associated targets with VENNY2.1 software, ${ }^{28}$ to identify potential SX therapeutic targets in RA. These targets were entered into the String Version 10.5 database (https://string-db.org/) ${ }^{28}$ to obtain the protein-protein interaction (PPI) diagram. The search was limited to human species, the protein interaction confidence score threshold was set to 0.4 , and disconnected nodes were hidden in the network representation. ${ }^{28}$ The PPI network was visualized using Cytoscape Version 3.6.1 software $^{28}$ to obtain the PPI diagram between the SX active components and RA-associated proteins. A Network Analyzer was used to analyze the network topology characteristics, including the degree centrality, betweenness centrality, and closeness centrality, and to identify core nodes of the PPI network with a degree value greater than 2 -fold the average degree. Active ingredients from the four herbal medicines that meet the criteria of $\mathrm{OB} \geq 30 \%$ and DL $\geq 0.18$ and their RAassociated targets were selected and entered in Cytoscape 3.6.1 to draw a network diagram of the SX active compounds and their therapeutic targets for RA.

GO enrichment and KEGG pathway enrichment analyses of key targets were conducted using the David 6.8 database (http://www.david.niaid.nih.gov). ${ }^{34}$ For GO analysis, biological process (BP), cell composition (CC) and molecular function (MF) were selected. KEGG pathway enrichment analysis used $\mathrm{P}<0.05$ as the cut-off value and the top 20 key pathways linked to arthritis were retained. ${ }^{37}$

\section{In silico Molecular Docking}

The pretreatment of ligands and target proteins was carried out by sybyl-x2.0. Pretreatment of ligands consisted of Gasteiger-Huckel charges to small molecules, maximum number of optimization iterations was set at 10,000, with a gradient convergence value of $0.005 \mathrm{kcal} /\left(\mathrm{mol}^{*} \mathrm{~A}\right)$, and with application of Powell's energy optimization algorithm; all other values were set to default parameters. Pre-treatment of target proteins consisted of removal of crystal water from all proteins, hydrogenation, removal of co-crystal ligand molecules occupying the binding sites, addition of missing amino acid residues and terminal residues, and application of Kollman United/Kollman all-atom force field and Assisted Model Building with Energy Refinement (AMBER) charges to minimize the protein. AutoDockTools (ADT) was used to 
generate pdbqt files, and finally AutoDock-vina was used to calculate nine favored binding conformations. The binding energy between each ligand and its target with the highest score, ie, presenting with the conformation with the strongest binding capacity, was calculated. PyMoL software was used for visualization. ${ }^{38}$ In this experiment, the Molaical ${ }^{39}$ program was used to calculate the Lipinski rule of five parameters of these compounds. In order to meet the requirements of this program, all compounds should be converted to mol2.dat file format and all parameters were set to their default values prior to calculation.

\section{Preparation of SX Miao Medicine Prescription}

A total of $2 \mathrm{~kg}$ of the four herbal medicines constitutive of SX at a JXT:DXT:FLZXGP:HGT weight ratio of 15:22:15:8 was boiled in $1000 \mathrm{~mL}$ of water and filtered with a gauze to obtain $500 \mathrm{~mL}$ of decoction. The herbal medicines were boiled again in $1000 \mathrm{~mL}$ of water and filtered with a gauze to obtain another $500 \mathrm{~mL}$ of decoction. The two $500-\mathrm{mL}$ decoctions were combined and boiled again until the volume was reduced to $500 \mathrm{~mL}$. This final solution was used as the test drug and stored in the refrigerator at $4{ }^{\circ} \mathrm{C}$ for up to 1 week. ${ }^{13-15}$ The final concentration of the drug corresponds to $4 \mathrm{~kg}$ of crude herbal medicine/L.

\section{Establishment of Collagen-Induced Arthritis (CIA) in Rat, Joint Scoring and Drug Administration}

All experimental procedures involving animals were approved by the Institutional Animal Care and Use Committee (IACUC) (Ethical number:1900868). The CIA model rat was established: Bovine type II collagen at the concentration of $2 \mathrm{mg} / \mathrm{mL}$ was mixed at equal volume with incomplete Freund's adjuvant on ice until uniformly and fully emulsified. 60 normal SD rats were injected with $200 \mu \mathrm{L}$ of emulsifier subcutaneously at the base of the tail, and 10 blank control rats were injected with the same amount of normal saline at the same site. One week later, a second dose of $100 \mu \mathrm{L}$ of emulsion was subcutaneously injected at the tail root to boost the immune response. The normal control group was injected with the same amount of normal saline subcutaneously at the tail root. The arthritis index (AI) score was determined to check for successful induction of arthritis. ${ }^{40}$ Fifty rats with successfully established CIA were randomly assigned to the SX high-dose group (SX $40 \mathrm{~g} / \mathrm{kg}$ group), SX medium-dose group (SX $20 \mathrm{~g} / \mathrm{kg}$ group), SX low-dose group (SX $10 \mathrm{~g} / \mathrm{kg}$ group), GTW-treated control group, and the model group (Mod) according to the random number table method. In addition, the control group (Nor) was established, which totaled 6 experimental groups, each with 10 animals.

After the last injection, the hind foot joints of the rats were measured once per week for AI scoring, ${ }^{13}$ which considered the redness, swelling, and joint deformities. ${ }^{41}$ Symptoms were given 0-4 scores as follows: 0 points (no redness or swelling), 1 point (red spots or mild joint swelling), 2 points (moderate redness and joint swelling), 3 points (moderate redness and joint swelling accompanied by mild dysfunction), and 4 points (severe swelling, stiffness and even joint deformity, and severe dysfunction). A score of 2 or more was considered successful RA development.

The volume of the hind feet of the rats was measured by water displacement every week. An average value of 3 measurements was used to calculate the degree of toe swelling. The swelling degree (ER) was calculated according to ER $(\%)=(\mathrm{Va}-\mathrm{Vb}) / \mathrm{Vb} \times 100 \%$ (where $\mathrm{Vb}$ is the volume before immersion, and $\mathrm{Va}$ is the volume after immersion). ${ }^{42}$ Two days after the successful establishment of CIA, the drug was given by gavage once a day for 21 consecutive days. The $\mathrm{SX} 40 \mathrm{~g} / \mathrm{kg}$ group, SX $20 \mathrm{~g} / \mathrm{kg}$ group,SX $10 \mathrm{~g} / \mathrm{kg}$ group were treated at different SX doses, GTW group was administered with GTW, and the blank and model groups were treated with saline. The CIA rats were anesthetized with $10 \%$ chloral hydrate on the day following the last drug administration. When the rats were under final anesthesia, the blood was collected by cardiac puncture and centrifuged to obtain serum for ELISA. The rats were killed by anesthetics overdose and placed on ice for collection of the inflamed hind legs. Part of the leg joints were fixed in $4 \%$ paraformaldehyde for $\mathrm{H} \& \mathrm{E}$ staining and the remaining leg joints were stored in a $-80^{\circ} \mathrm{C}$ freezer.

\section{Hematoxylin-Eosin Staining}

Rat hind leg ankle joints were fixed with $10 \%$ paraformaldehyde for $24 \mathrm{~h}$, rinsed in $0.2 \mathrm{M}$ phosphate buffer solution, and then decalcified in EDTA decalcification solution at room temperature. Decalcification was checked every day and the 
decalcification solution was replaced once a week for one month. Samples were then rinsed with distilled water for 25 min., dehydrated through a series of graded ethanol, cleared in xylenes, embedded in paraffin, cut into 3-5 $\mu \mathrm{m}$ tissue sections, and H\&E stained. The histomorphological and pathological changes of the ankle synovium were analyzed under a microscope. $^{43}$

\section{Measurement of the Serum Content of VEGF-A, IL-6, IL-2, TNF- $\alpha$ Protein}

After 24 hours, the final medication involved several steps, namely: 1) Anesthesia with $10 \%$ chloral hydrate solution $0.4 \mathrm{~mL} / 100 \mathrm{~g}$ intraperitoneal injection; 2) taking blood from the abdominal aorta; 3) after $2 \mathrm{~h}$ at room temperature, $3000 \mathrm{r} / \mathrm{min} ., 4^{\circ} \mathrm{C}$ Centrifugation (radius $13.5 \mathrm{~cm}$ ) for $10 \mathrm{~min} . ; 4$ ) the supernatant is kept in a $-80^{\circ} \mathrm{C}$ refrigerator; 5) serum VEGF-A, IL-6, IL-2, TNF- $\alpha$ levels in rats were measured by ELISA method. The operation was carried out strictly according to the instructions of the kit. VEGF-A, IL-6, IL-2, TNF- $\alpha$ levels in serum were calculated. $^{44}$

\section{Measurement of VEGF-A, PI3K, AKT, NF-кBp65, StartI, PTGS2 mRNA Level in Synovial Tissues of CIA Rats by qRT-PCR}

Trizol was used to extract the total RNA from the rat synovial tissues. One microgram of the total RNA was used for reverse transcription. Primers were designed according to the sequence of rat VEGF, PI3K, AKT, NF- $\kappa \mathrm{B}$, STAT1, PTGS2 and GAPDH (Table 1). The PCR reactions were carried out according to the instructions provided in the used qPCR reaction kit (Baori Biotechnology, Beijing, China). The reaction conditions were 5 min. of denaturation at $95^{\circ} \mathrm{C}$, followed by 40 cycles of $95^{\circ} \mathrm{C}$ for $10 \mathrm{sec}$., and $60^{\circ} \mathrm{C}$ for $30 \mathrm{sec}$; melting curves were obtained by heating the reaction at $95^{\circ} \mathrm{C}$ for $15 \mathrm{sec}$., $60^{\circ} \mathrm{C}$ for $60 \mathrm{sec}$, and $95^{\circ} \mathrm{C}$ for $15 \mathrm{sec}$. The results were calculated by the $2^{-\Delta \Delta \mathrm{CT}}$ method. $^{45}$

Table I List of Primer Sequences Used for qRT-PCR Analysis in This Study

\begin{tabular}{|c|c|c|c|c|c|}
\hline Gene Name & $\begin{array}{l}\text { Sequence } \\
\text { of Primer }\end{array}$ & $\begin{array}{l}\text { Fragment } \\
\text { Length (bp) }\end{array}$ & $\begin{array}{l}\text { Annealing } \\
\text { Temperature }\left({ }^{\circ} \mathrm{C}\right)\end{array}$ & $\begin{array}{l}\text { Accession } \\
\text { Numbers }(\mu L)\end{array}$ & $\begin{array}{l}\text { Average } \\
\text { Efficiencies }\end{array}$ \\
\hline VEGF-A-F & 5'-CCTTAGGTGCTTCCCCATATC-3' & $132 \mathrm{bp}$ & $60^{\circ} \mathrm{C}$ & 0.4 & \multirow[t]{2}{*}{$100 \%$} \\
\hline VEGF-A-R & 5'-GAGCCCGCATTCTCGTTC-3' & $132 \mathrm{bp}$ & $60^{\circ} \mathrm{C}$ & 0.4 & \\
\hline$P I 3 K-F$ & 5'-CAATACTTGATGTGGCTGACG-3' & $139 \mathrm{bp}$ & $60^{\circ} \mathrm{C}$ & 0.4 & \multirow[t]{2}{*}{$98 \%$} \\
\hline$P I 3 K-R$ & 5'-CTTCCCTCGCAATAGGTTCT-3' & $139 b p$ & $60^{\circ} \mathrm{C}$ & 0.4 & \\
\hline AKT-F & 5'-CTTCTATGGTGCGGAGATTG-3' & $134 \mathrm{bp}$ & $60^{\circ} \mathrm{C}$ & 0.4 & \multirow[t]{2}{*}{$99 \%$} \\
\hline$A K T-R$ & 5'-ACAGCCCGAAGTCCGTTA-3' & $134 \mathrm{bp}$ & $60^{\circ} \mathrm{C}$ & 0.4 & \\
\hline$N F-\kappa B$ p $65-F$ & 5'-CATACGCTGACCCTAGCCTG-3' & $138 \mathrm{bp}$ & $60^{\circ} \mathrm{C}$ & 0.4 & \multirow[t]{2}{*}{$99 \%$} \\
\hline$N F-\kappa B p 65-R$ & 5'-TTTCTTCAATCCGGTGGCGA-3' & $138 \mathrm{bp}$ & $60^{\circ} \mathrm{C}$ & 0.4 & \\
\hline STATI-F & 5'-ACTTGACCTTGCTAGTGGTATGT-3' & $127 \mathrm{bp}$ & $60^{\circ} \mathrm{C}$ & 0.4 & \multirow[t]{2}{*}{$100 \%$} \\
\hline STATI-R & 5'-CTTTGCTGAGGACCTGTAGTCTGAG-3' & $127 \mathrm{bp}$ & $60^{\circ} \mathrm{C}$ & 0.4 & \\
\hline PTGS2-F & 5'-GGTTGATTTCTCCAGCATTTC-3' & $14 \mathrm{Ibp}$ & $60^{\circ} \mathrm{C}$ & 0.4 & \multirow[t]{2}{*}{$99 \%$} \\
\hline PTGS2-R & 5'-AGGCTCTACTTTGATCGCACT-3' & $14 \mathrm{Ibp}$ & $60^{\circ} \mathrm{C}$ & 0.4 & \\
\hline GAPDH-F & 5'-GAAGCTGGTCATCAACGGGA-3' & $125 \mathrm{bp}$ & $60^{\circ} \mathrm{C}$ & 0.4 & \multirow[t]{2}{*}{$100 \%$} \\
\hline GAPDH-R & 5'-GGCGGAGATGATGACCCTTT-3' & $125 \mathrm{bp}$ & $60^{\circ} \mathrm{C}$ & 0.4 & \\
\hline
\end{tabular}




\section{Quantification of PI3K, AKT, p-AKT, NF-אBp65, p-NF-кBp65 Protein Levels in Synovial Tissues by Western Blot}

Rat synovial tissues were ground in liquid nitrogen and the protein concentration was measured using a Bicinchoninic

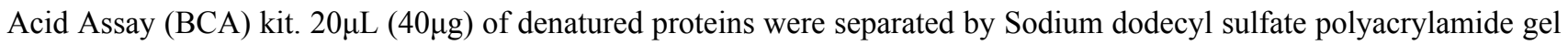
electrophoresis (SDS-PAGE) and transferred to a PVDF membrane. The membrane was blocked overnight with 5\% skimmed milk and incubated with the primary antibodies: anti-beta-actin diluted at 1:7000 at room temperature for $2 \mathrm{~h}$, or anti-PI3K, AKT, p-AKT, NF-кBp65, p-NF-KBp65 antibodies diluted at 1:1000, at $4{ }^{\circ} \mathrm{C}$ overnight. After 3 washes of 10 min. in TBST (pH 7.5, Tris- $\mathrm{HCl} 10 \mathrm{mM}, \mathrm{NaCl} 150 \mathrm{mM}$, Tween-20 0.05\%), the membrane was incubated with anti-rabbit (1:1000) or anti-mouse (1:5000) secondary antibodies at room temperature for $90 \mathrm{~min}$. The membrane was then washed in $1 \times$ TBST, developed and analyzed on a gel imager. ${ }^{46,47}$

\section{Statistical Analysis}

Statistical analyses were performed using SPSS software version 22.0 (Armonk city, New York state, USA). Unless otherwise noted, all data are presented as mean \pm standard error of mean (SEM). Shapiro-Wilk test and Q-Q plots were used to assess data normality. Normally distributed data were analyzed using one-way analysis of variance (ANOVA), and the Kruskal-Wallis test was used if the data did not pass the normality test. The post-hoc test of Kruskal-Wallis test was Dunn's pairwise comparison test, with p-values adjusted by Bonferroni correction for multiple comparisons. If the results from the ANOVA tests were statistically significant, the Fisher's least significant difference (LSD) tests were used to conduct pairwise comparison. Comparisons with $p$ values $<0.05$ were considered statistically significant. ${ }^{43}$

\section{Results}

\section{Active Compounds Identified in SX and Their Putative Targets}

$\mathrm{OB}$ is an important parameter of drug absorption and metabolism. The higher the value, the better the biological activity and drug-like DL of the drug. Therefore, a standard $\mathrm{OB} \geq 30 \%$ and a DL $\geq 0.18$ are commonly used in the literature as the selection criteria for active ingredient screening. TCMSP(https://old.tcmsp-e.com/tcmsp.php), Traditional Chinese Medicine and Chemical Composition Database(http://www.organchem.csdb.cn/scdb/default.htm?nCount=13303008), and PubMed(https://pubmed.ncbi.nlm.nih.gov/?term=) were searched for the chemical components of the four traditional Chinese medicines in SX. After duplications were removed and the low confident hits eliminated by a cut-off score of $30 \%$ for $\mathrm{OB}$ and 0.18 for DL, a total of 33 active compounds were identified, including 5 compounds from JXT (15.15\%), 11 from DXT (33.33\%), 13 from FLZXGP (39.39\%), and 4 from HGT (12.12\%) (Table 2). The network diagram for the active components of Chinese herbal medicine was established (Figure 1), and the triangle (or yellow) represents the drug, and the circle represents the active ingredient (blue individual drug ingredient, green represents the intersecting active ingredient). In the network diagram, According to the degree of value, JXT and FLZXGP play a major role in the compound SX, and the first three active compounds were $\beta$-sitosterol (+)-catechin, ent-Epicatechin (Figure 1 or Supplementary Table S1). In addition, a total of 457 putative protein targets of these compounds were identified using the TCMSP and the Swiss Target Predication databases.

\section{Prediction of SX Molecular Targets for the Treatment of RA}

The RA-related target genes were screened from the TTD (http://db.idrblab.net/ttd/), Drugbank (https://www.drugbank. ca/), and DisGeNE databases (https://www.disgenet.org/). Using the keyword "Rheumatoid arthritis" to search the databases, 306 associated genes were identified. From the comparative analysis between the 306 RA-related and 457 putative SX targets, 44 were predicted as SX targets for the treatment of RA (Figure 2, Supplementary Table S2). 44 targets were entered into the String Version 10.5 database, and then the Cytoscape Version 3.6.1 software was used for visualization processing to obtain the protein interaction (PPI) network diagram of the SX treatment of RA (Figure 3). The circle represents the target point. The color of the target point gradually changes according to the degree value. The larger the degree value, the larger the circle.The degree value is from large to small, and the color gradually changes from 
Table 2 Active Compounds in the Four Herbal Medicines Constitutive of SX

\begin{tabular}{|c|c|c|c|c|}
\hline Herbal Medicine & MOL ID & Molecule Name & OB (\%) & DL \\
\hline Sargentodoxa cuneata (Oliv.) & MOL000358 & $\beta$-sitosterol & 36.91 & 0.75 \\
\hline \multirow[t]{4}{*}{ Rehd. et Wils. (DXT) } & MOL000492 & $(+)$-catechin & 54.83 & 0.24 \\
\hline & MOL000073 & ent-epicatechin & 48.96 & 0.24 \\
\hline & MOL006504 & $(-)$-catechin gallate & 53.57 & 0.75 \\
\hline & MOL00I792 & Liquiritigenin & 32.76 & 0.18 \\
\hline \multirow[t]{11}{*}{ Toddalia asiatica L. Lam. (FLZXGP) } & MOL00I46I & Dihydrochelerythrine & 32.73 & 0.81 \\
\hline & MOL00233I & $\mathrm{N}$-methylflindersine & 32.36 & 0.18 \\
\hline & MOL000358 & $\beta$-sitosterol & 36.91 & 0.75 \\
\hline & MOL002663 & Skimmianine & 40.14 & 0.2 \\
\hline & MOL0I080I & $\beta$-amyrin & 32.68 & 0.3 \\
\hline & MOL005I05 & Oxychelerythrine & 44.22 & 0.84 \\
\hline & MOL007930 & Hesperidin & 47.74 & 0.27 \\
\hline & MOL005I00 & Hesperetin-7-O-rutinoside & 47.74 & 0.27 \\
\hline & MOL0I0425 & Oxyavicine & 53.09 & 0.87 \\
\hline & MOL002666 & Chelerythrine & 34.18 & 0.78 \\
\hline & MOL002644 & Phellopterin & 40.19 & 0.28 \\
\hline \multirow[t]{14}{*}{ Spatholobus suberectus Dunn (JXT) } & MOL000502 & Cajanin & 68.8 & 0.27 \\
\hline & MOL000497 & Licochalcone A & 40.79 & 0.29 \\
\hline & MOL000358 & $\beta$-sitosterol & 36.91 & 0.75 \\
\hline & MOL000503 & Medicagol & 57.49 & 0.6 \\
\hline & MOL000392 & Formononetin & 69.67 & 0.21 \\
\hline & MOL000469 & 7-oxo- $\beta$-sitosterol & 40.93 & 0.78 \\
\hline & MOL000492 & $(+)$-catechin & 54.83 & 0.24 \\
\hline & MOL000073 & ent-epicatechin & 48.96 & 0.24 \\
\hline & MOL000004 & Procyanidin $\mathrm{BI}$ & 67.87 & 0.66 \\
\hline & MOL002565 & Medicarpin & 49.22 & 0.34 \\
\hline & MOL009009 & $(+)$-medioresinol & 87.19 & 0.62 \\
\hline & MOL004576 & Taxifolin & 57.84 & 0.27 \\
\hline & MOL004917 & Glycyroside & 37.25 & 0.79 \\
\hline & MOL0004I7 & Calycosin & 47.75 & 0.24 \\
\hline \multirow[t]{4}{*}{ Periploca forrestii Schltr. (HGT) } & MOL000087 & $\beta$-sitosterol-3-O- $\beta$-D-glucopyranoside & 36.91 & 0.75 \\
\hline & MOL005658 & Periplogenin & 36.61 & 0.74 \\
\hline & MOL000357 & Eleutheroside A & 36.91 & 0.75 \\
\hline & MOL000357 & Elexandrin & 36.91 & 0.75 \\
\hline
\end{tabular}

Abbreviations: SX, Sidaxue; JXT, ji-xue-teng; FLZXGP, fei-long-zhang-xue-geng-pi; DXT, da-xue-teng; HGT, hei-gu-teng; OB, oral bioavailability; DL, drug-like properties.

orange to green (at the same time, the graph is divided into 3 levels, the inner layer to the middle layer to the outer layer represents the degree value from large to small). The figure includes 48 nodes and 237 edges, where the node represents the target gene, and the edge represents the line connecting the node, the same node is passed by multiple lines, indicating that the more important. The average degree of freedom is 9.875 . There are 6 targets that are more than twice the average degree of freedom. There are TNF, IL6, VEGF-A, CXCL8, PTGS2, IL2 in descending order of degrees of freedom (Table 3, Figure 3).(See Supplementary Table S3 for other target point degree values.)

\section{Network Diagram Built from SX Active Compounds and RA-Associated Targets}

The SX-compounds-RA PPI network included 242 nodes and 743 edges (Figure 4). The inverted triangle represents the drug, the oblique square represents the component, and the circle represents the target. The color and size of the graph gradually changed from deep to shallow (Or from orange to green) and large to small according to the degree of freedom. The larger the degree value was, the more important role the part played in the PPI. The average node degree was 6.14. According to the degree of freedom, it can be seen that the blood root bark of JXT and FLZXGP are the main drugs, and 


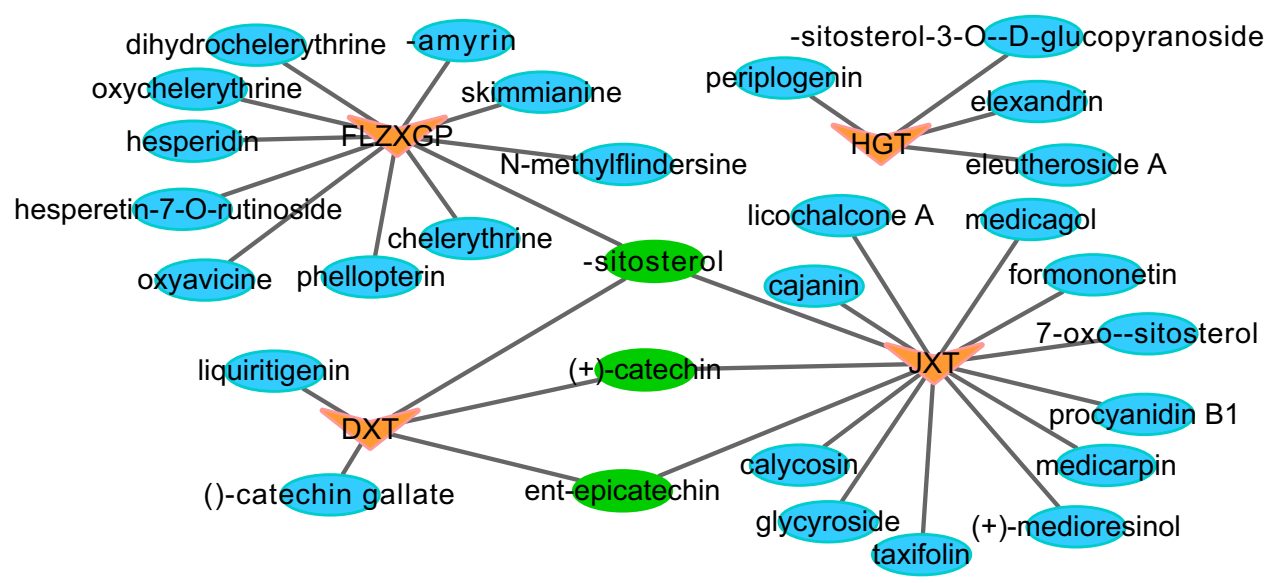

Figure I The network construction for herbs-active ingredient.

Notes: In Figure I, the triangle (or yellow) represents the drug, and the circle represents the active ingredient (blue individual drug ingredient, green represents the intersecting active ingredient).

\section{Ingredient targets}

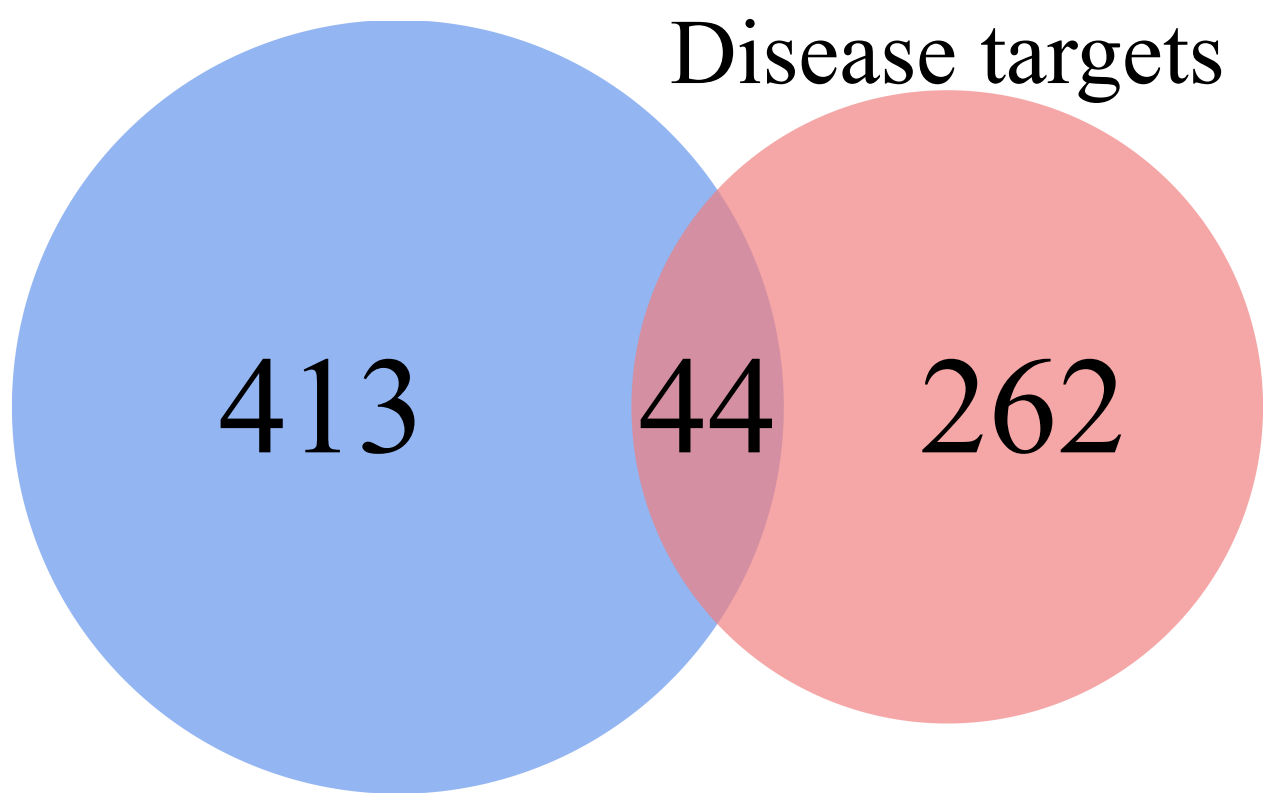

Figure 2 Venn diagram of SX drug targets and RA-disease proteins.

the top 10 ingredients and targets are sorted (Table 4 and Figure 4). The key ingredients include MOL000358 ( $\beta$ sitosterol), MOL002565 (Medicarpin), MOL000469 (7-oxo- $\beta$-sitosterol), MOL002663 (skimmianine), MOL000392 (formononetin), MOL000497 (licochalcone A), key targets include PTGS1, ESR1, CA2, PTPN1, CYP19A1, ESR2, CA12, ABCB1, CA1,CA7.

\section{GO and KEGG Pathway Enrichment Analysis of the Putative Targets of SX in RA Treatment}

DAVID software was used for the enrichment analysis of the 44 targets. By choosing a threshold $p$ value $<0.05$, this analysis revealed 479 GO biological processes and 22 KEGG signal pathways involving SX target proteins. The resulting 


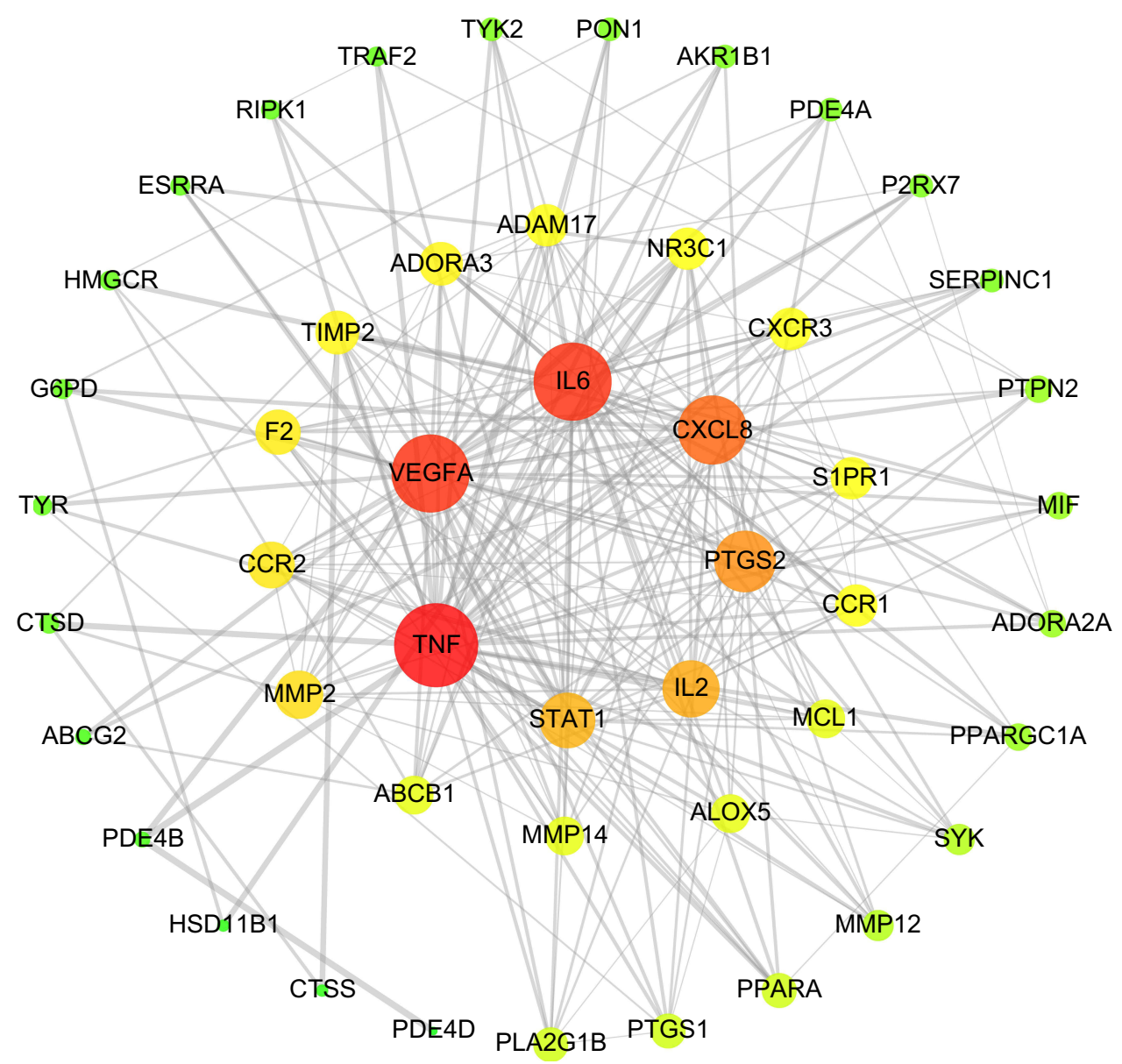

Figure 3 PPI network of RA targets of SX.

Notes: The circle represents the target point. The color of the target point gradually changes according to the degree value. The larger the degree value, the larger the circle. The degree value is from large to small, and the color gradually changes from orange to green (at the same time, the graph is divided into 3 levels, the inner layer to the middle layer to the outer layer represents the degree value from large to small).

GO biological processes and KEGG pathways were further analyzed to infer potential biological functions to SX targets, with a threshold $\mathrm{P}$ value $<0.05$.

In the GO function analysis (Figure 5), the top 10 items of biological function are listedon the vertical axis, including molecular function(MF), cell composition(CC), and biological process(BP), the horizontal axis in the figure represents the statistical $P$ value of biological functions. In Figure 5, blue represents MF, green represents CC, and pink represents BP. Among them, molecular function (MF) mainly includes lipid binding, protein dimerization activity, and protein homodimerization activity, endopeptidase activity, cytokine activity, peptidase activity, calcium ion binding, identical protein binding, identical protein binding, identical protein binding. From the MF-target map (Figure 6), we can see that

Table 3 Key Nodes and Topological Parameters of the PPI Network

\begin{tabular}{|l|l|l|l|}
\hline Gene & Degree & Betweenness Centrality & Closeness Centrality \\
\hline TNF & 38 & 0.25 & 0.84 \\
IL-6 & 34 & 0.14 & 0.77 \\
VEGF & 34 & 0.18 & 0.78 \\
IL-8 & 28 & 0.08 & 0.70 \\
PTGS2 & 23 & 0.04 & 0.65 \\
IL-2 & 20 & 0.03 & 0.62 \\
\hline
\end{tabular}

Abbreviation: PPI, protein-protein interaction. 


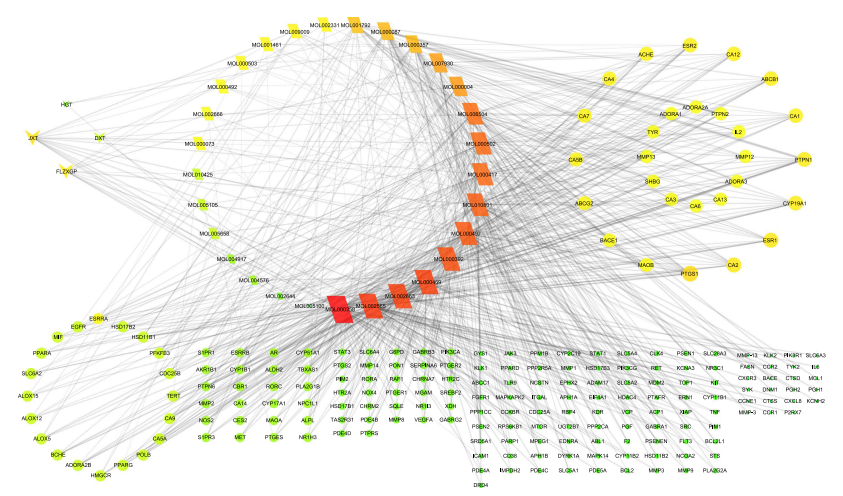

Figure 4 SX-compounds-RA PPI network.

Notes: The inverted triangle represents the drug, the oblique square represents the component, and the circle represents the target. The color and size of the graph gradually changed from deep to shallow (Or from orange to green) and large to small according to the degree of freedom. The larger the degree value was, the more important role the part played in the PPI.

the triangle represents MF, the circle represents the target point, and the gray line represents the degree value of the node, the degree value from large to small follows a gradient from orange to green. The more lines, the greater the degree value which in turn indicates the greater the biological importance. The key genes enriched include MMP14, MMP2, MMP12, PON1, VEGF-A, F2, ADAM17, CTSS, IL6, IL2, TNF, PTGS2, PPARA, MIF.

Cell composition (CC) mainly affects the cell fraction, plasma membrane, extracellular area, extracellular space and so on. From the CC-target diagram (Figure 7), we can see that the triangle represents the CC, the circle represents the

Table 4 Top Network Topology Parameters of the Interaction Network Between SX Herbal Medicines, Active Compounds and RA-Associated Targets

\begin{tabular}{|l|l|l|l|}
\hline Name & Degree & Betweenness Centrality & Closeness Centrality \\
\hline JXT & 14 & 0.03 & 0.41 \\
FLZXGP & $1 \mathrm{l}$ & 0.02 & 0.35 \\
DXT & 4 & 0.00 & 0.32 \\
HGT & 3 & 0.00 & 0.26 \\
MOL000358 & 69 & 0.18 & 0.43 \\
MOL002565 & 58 & 0.16 & 0.41 \\
MOL000469 & 56 & 0.13 & 0.41 \\
MOL002663 & 56 & 0.14 & 0.41 \\
MOL000392 & 52 & 0.08 & 0.41 \\
MOL000497 & 50 & 0.13 & 0.40 \\
MOL0I080I & 48 & 0.12 & 0.37 \\
MOL000502 & 46 & 0.07 & 0.40 \\
MOL0004I7 & 46 & 0.07 & 0.40 \\
MOL006504 & 44 & 0.08 & 0.37 \\
PTGSI & 16 & 0.04 & 0.46 \\
ESRI & 12 & 0.02 & 0.43 \\
CA2 & 12 & 0.02 & 0.43 \\
PTPNI & 11 & 0.08 & 0.45 \\
CYPI9AI & 11 & 0.03 & 0.45 \\
ESR2 & 10 & 0.01 & 0.42 \\
CAI2 & 10 & 0.01 & 0.42 \\
ABCBI & 10 & 0.02 & 0.43 \\
CAI & 10 & 0.01 & 0.42 \\
CA7 & 9 & 0.01 & 0.38 \\
\hline Notes & & & \\
\hline
\end{tabular}

Notes: The table is arranged according to herbs, compounds, and targets. The higher the degree value, the more important it is.

Abbreviations: SX, Sidaxue; RA, Rheumatoid arthritis; JXT, ji-xue-teng; FLZXGP, fei-long-zhang-xue-geng-pi; DXT, da-xue-teng; HGT, hei-gu-teng. 


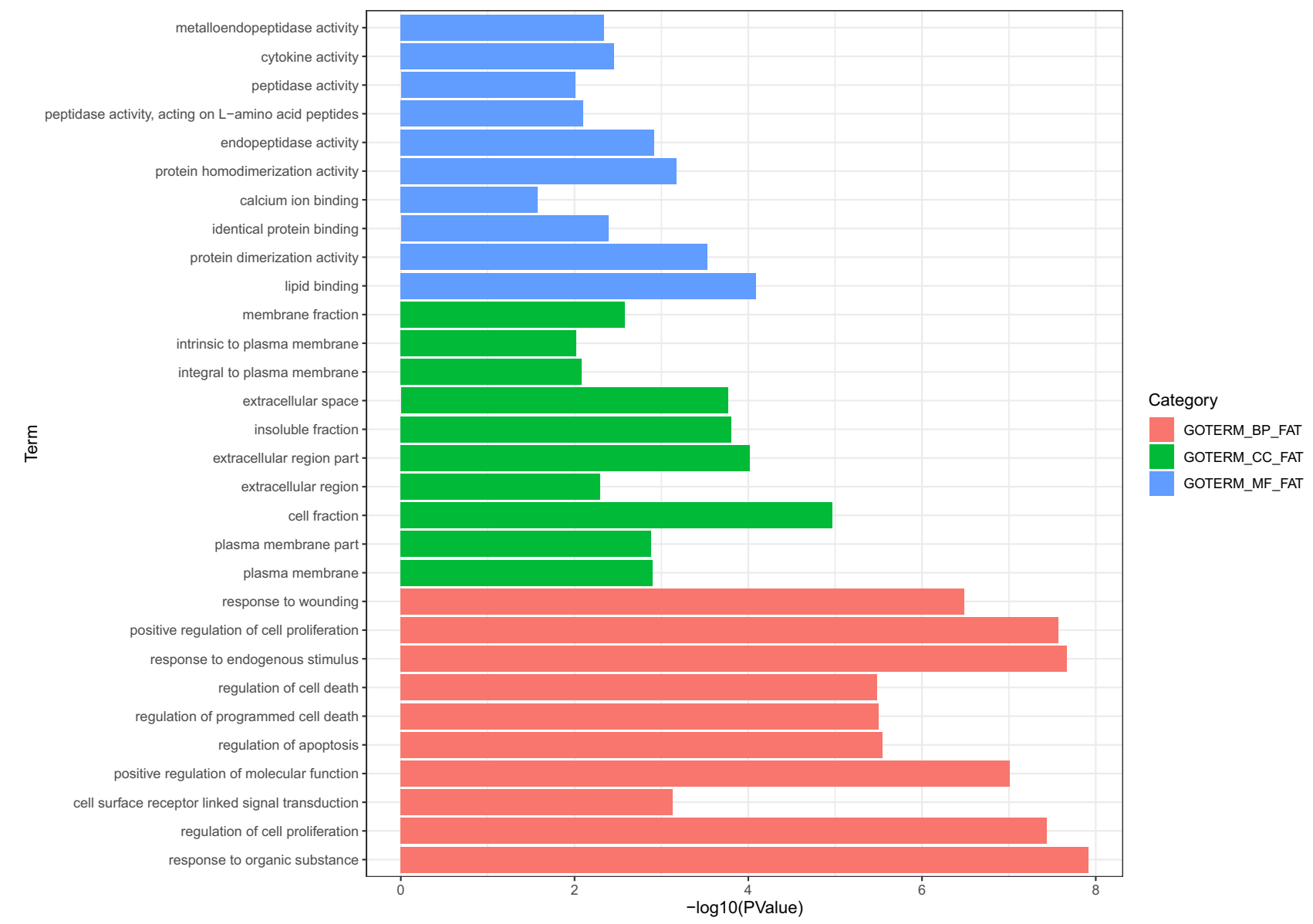

Figure 5 Gene ontology analysis of key target genes.

Notes: In Figure 5, the top 10 items of biological function are listed on the vertical axis, including molecular function (MF, blue), cell composition (CC, green), and biological process (BP, pink), the horizontal axis in the figure represents the statistical $\mathrm{P}$ value of biological functions.

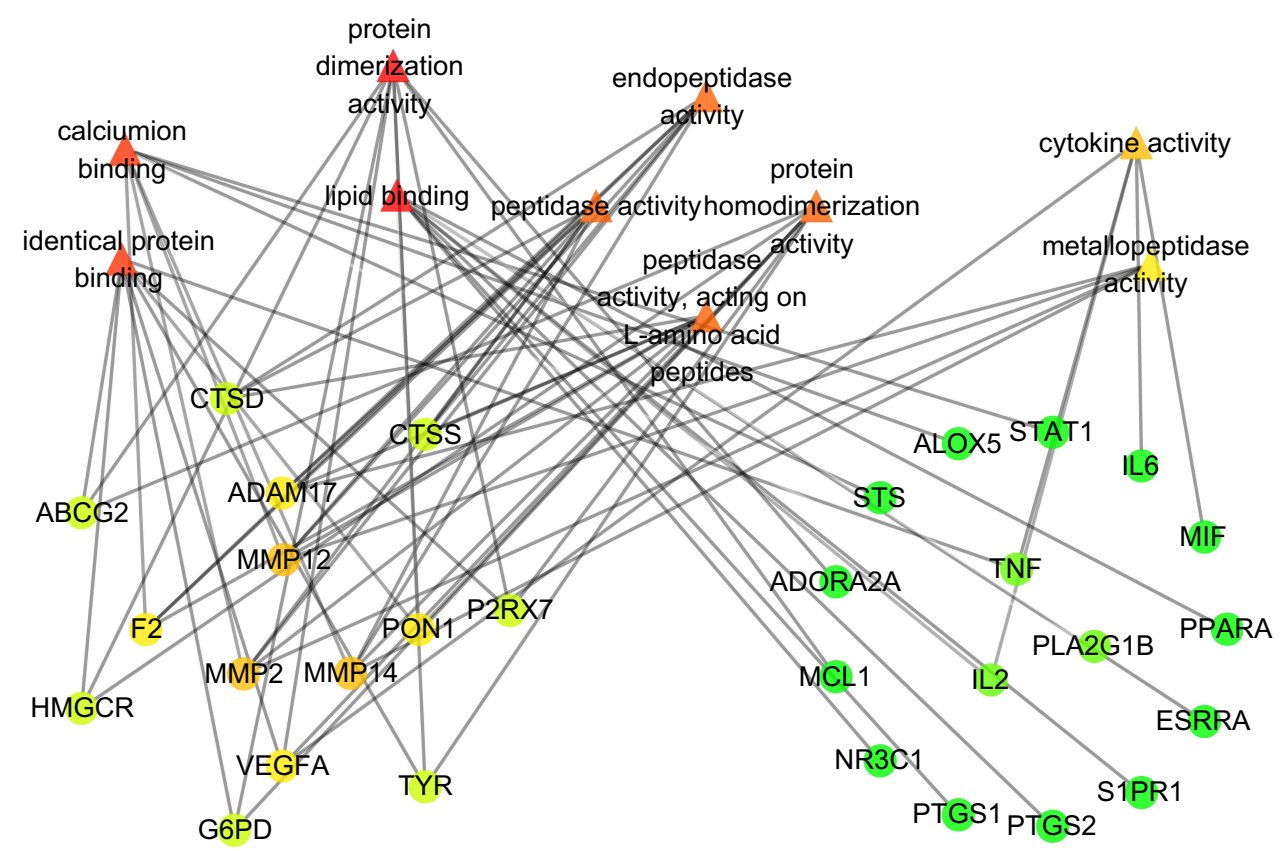

Figure 6 PPI network of key targets of MF.

Notes: The circle represents the target point, and the gray line represents the degree value of the node, the degree value from large to small follows a gradient from orange to green. The more lines, the greater the degree value which in turn indicates the greater the biological importance. 


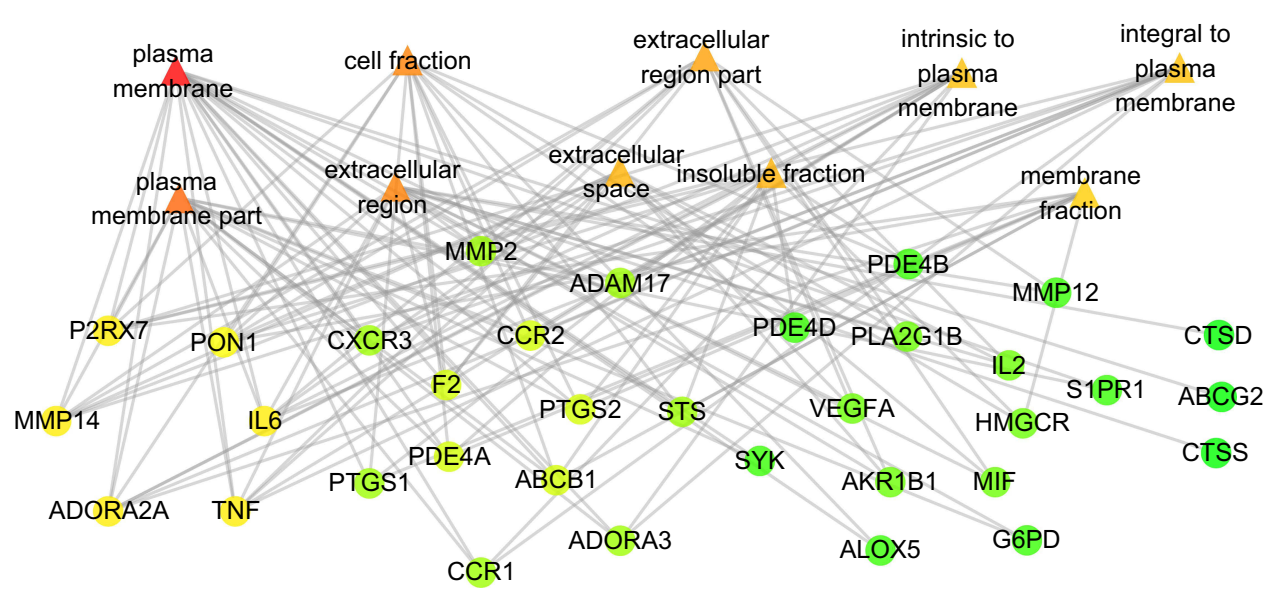

Figure 7 PPI network of key targets of CC.

Notes: The circle represents the target point, and the gray line represents the degree value of the node, the degree value from large to small follows a gradient from orange to green. The more lines, the greater the degree value which in turn indicates the greater the biological importance.

target point, and the gray line represents the degree value of the node, the degree value from large to small follows a gradient from orange to green. The more lines and the greater the degree value, the greater its biological importance. According to the degree value, it can be seen that the key genes enriched include IL2, TNF, ADORA2A, MMP14, PTGS2, PON1, CCR1, VEGFA, ALOX5, MMP12, PDE4B.

The biological process (BP) is mainly the response to organic substances, the regulation of cell proliferation, the response to endogenous stimulus, the positive regulation of cell proliferation, the regulation of apoptosis, cell surface receptor linked signal transduction, and positive regulation of molecular function, regulation of programmed cell death, regulation of cell death, response to wounding. From the BP-target map (Figure 8), we can see that the triangle represents the BP, the circle represents the target point, and the gray line represents the degree value of the node, the degree value from large to small follows a gradient from orange to green. The more lines, the greater the degree value indicates the greater its biological importance. According to the degree value, it can be seen that the key genes enriched include

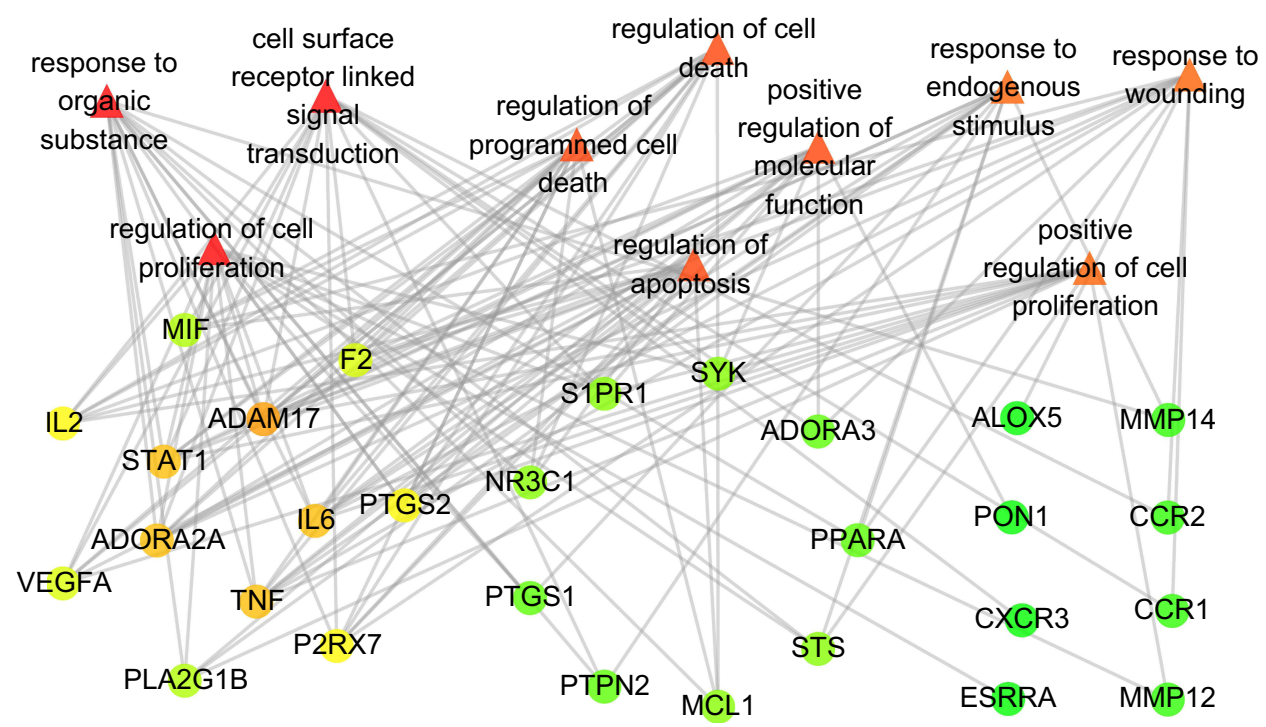

Figure 8 PPI network of key targets of BP.

Notes: The circle represents the target point, and the gray line represents the degree value of the node, the degree value from large to small follows a gradient from orange to green. The more lines, the greater the degree value which in turn indicates the greater the biological importance. 
ADAM17, IL6, TNF, ADORA2A, STAT1, PTGS2, F2, MIF, P2RX7, PPARA, PTPN2, CCR1, CCR2.(For details of GO analysis, see Supplementary Table S4).

KEGG enrichment results in 22 KEGG pathways, which are related to the immune response, inflammation, viral infection, cancer, etc. The top 20 KEGG signaling pathways are selected for mapping (Figure 9). For the percentage, the area of the bubble represents the number of enriched genes in the pathway, and the color of the bubble represents the size of the $\mathrm{P}$ value. The signal pathways in the figure include Sphingolipid signaling pathway, Neuroactive ligand-receptor interaction, PI3K-AKT signaling pathway, cAMP signaling pathway, TNF signaling pathway, NF- $\mathrm{KB}$ signaling pathway, Tuberculosis, NOD-like receptor signaling pathway, Intestinal immune network for IgA production, etc. In the KEGGtarget diagram (Figure 10), the triangles represent Pathway, the circle represents the target, the target in the figure is gradient from orange to green according to the degree value from large to small. The more lines, the greater the degree value indicates the greater its biological importance. According to the degree value, the key genes enriched include IL6, TNF, and VEGF- A, IL2, PTGS2, PI3K, AKT, NF-кB p65, CASP1, ADORA3, CTSD, PTGS1, MCL1, ALOX5.(The remaining pathway-enriched genes are shown in Supplementary Table S5).

\section{Molecular Docking Analysis}

To further document the potential effect of the 13 main active compounds of SX on the 9 key hypothetical targets, including TNF- $\alpha$, VEGF-A, VEGFR-2, IL-6, PTGS1, IL-2, NF-кB, AKT, and PI3K, we performed a molecular docking analysis in silico. The binding free energies are shown in Table 5. The free energy of binding between compound $\beta$ amyrin, cajanin, (-)-catechin gallate, eleutheroside A, calycosin, $\beta$-sitosterol-3-O- $\beta$-D-glucopyranoside, and ormononetin, and the target proteins TNF- $\alpha$, VEGF-A, IL-2, AKT, and PI3K was less than the free energy of binding between these

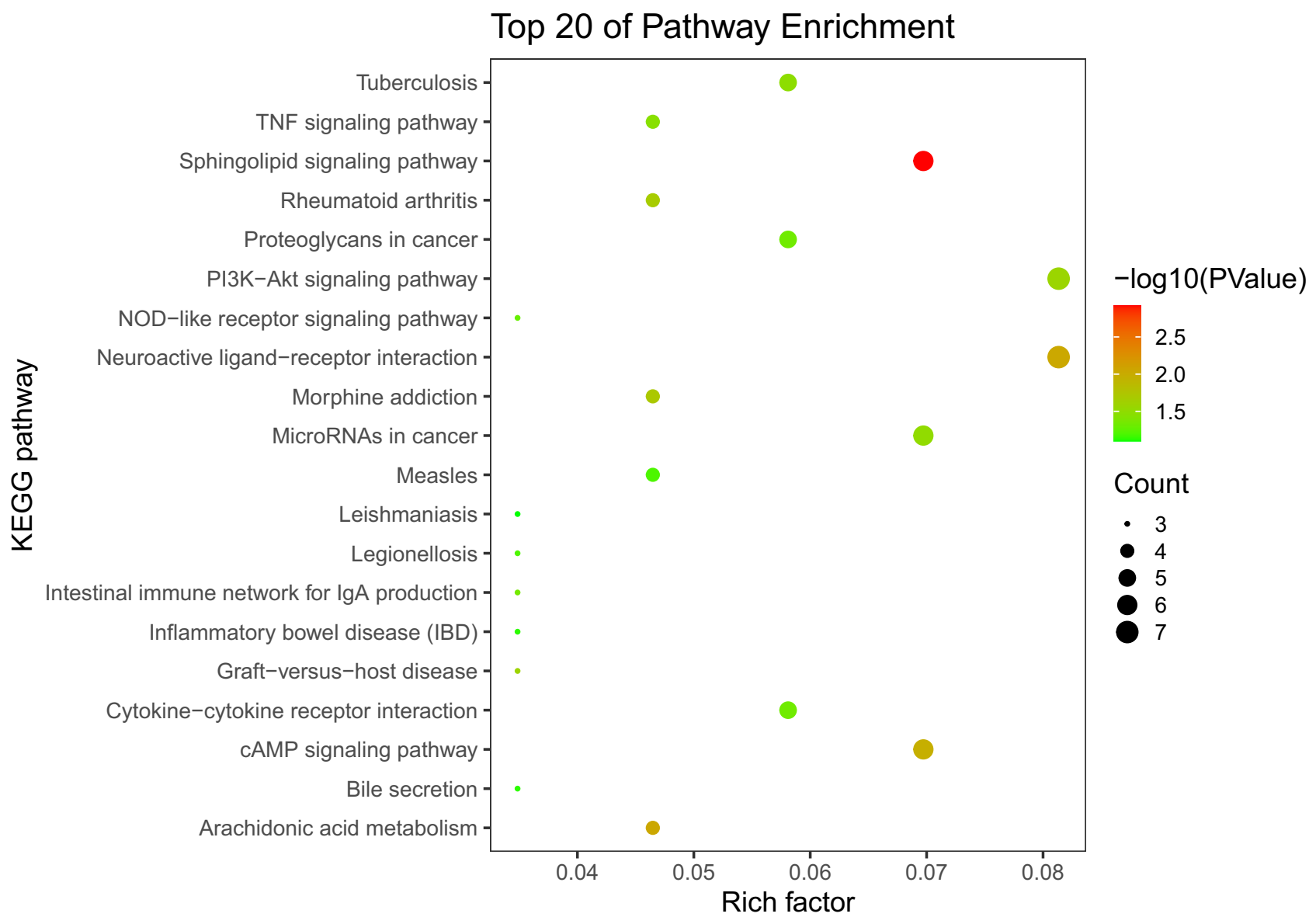

Figure 9 Top 15 pathways by KEGG analysis.

Notes: In Figure 9, the area of the bubble represents the number of enriched genes in the pathway, and the color of the bubble represents the size of the $\mathrm{P}$ value. 


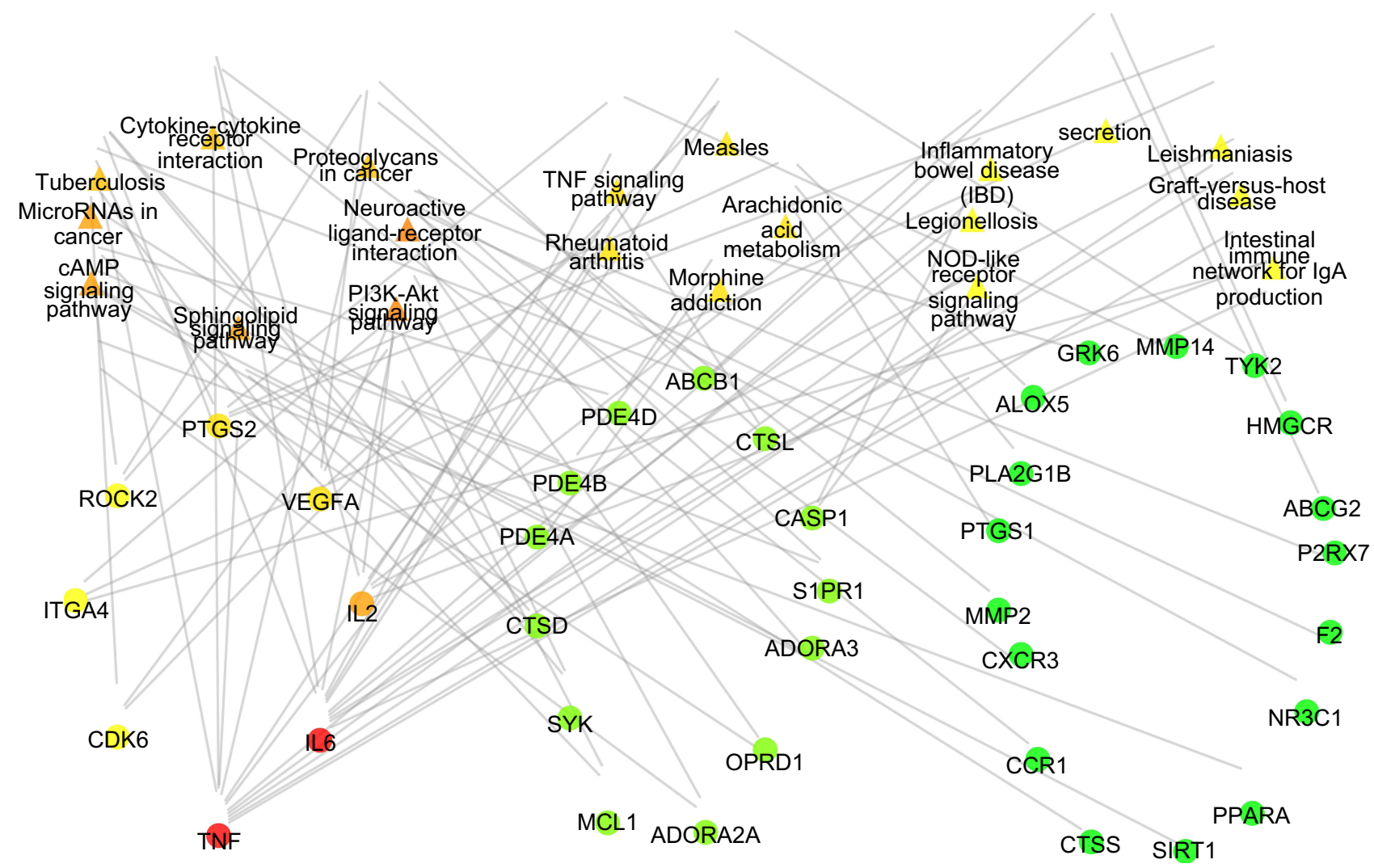

Figure 10 PPI network of key targets of KEGG analysis.

Notes: the triangles represent Pathway, the circle represents the target, the target in the figure is gradient from orange to green according to the degree value from large to small. The more lines, the greater the degree value indicates the greater its biological importance.

targets and their original ligands, suggesting that these compounds are the key pharmacological components of SX for RA treatment. The free energy of binding between the compounds $\beta$-sitosterol, eleutheroside A, medicarpin, and cajanin and the targets VEGFR-2, PTGS, and NF- $\mathrm{kB}$ was close to the free energy of the binding between these targets and their original ligands. IL-6 had a high compatibility with licochalcone A and cajanin. These results imply that treatment with SX may affect TNF- $\alpha$, VEGF-A, VEGFR-2, IL-6, NF- $\kappa$ B, AKT, and PI3K in RA patients. The target proteins and the small molecules with strong binding affinity were visualized by PyMoL software (Figure 11). Docking results show that the binding between compounds and the target proteins is mainly mediated by hydrophobic interactions and especially hydrogen bonds. The Lipinski rule calculation results show that most of the calculated molecules satisfy the RO5 criterion. However, there are still a few molecules that are beyond the limits of RO5. The relative molecular weight values of MOL000087 and MOL000357 are greater than 500, which exceeds the ideal range of RO5, furthermore, the number of hydrogen bond acceptors of these two molecules exceeds 5, and the number of rotating bonds is too large, causing them to be potentially overly flexible, which adversely affects their drugability. In addition, their excessive ClogP values are also detrimental to the dissolution of these two drugs in the aqueous environment. (For Lipinski rule data of compounds involved in molecular docking, see Supplementary Table S6).

\section{Assessment of SX Effect of on the Joint Swelling and Arthritis Score of CIA Rats}

In order to determine whether the CIA model was successfully established before treatment and assessment of drug efficacy, we established a cohort of CIA rats and compared disease evolution in groups of these animal treated either with different doses of SX, with GTW, another herbal medicine used for its anti-arthritis properties, or left untreated. The AI score and foot swelling were determined. Before treatment, the joints of hind limbs of CIA rats all showed redness and swelling to varying degrees. The redness and swelling of the knees, ankles, and interdigital joints of the 
Table 5 Docking Scores of the 13 Candidate Compounds and 9 Key Targets

\begin{tabular}{|c|c|c|c|c|c|c|c|c|c|c|c|c|}
\hline \multirow[t]{2}{*}{ MOL ID } & \multirow[t]{2}{*}{ Molecula Name } & \multicolumn{11}{|c|}{ Molecular Docking Score } \\
\hline & & $\begin{array}{l}\text { Gene Name } \\
\text { (PDB ID) }\end{array}$ & $\begin{array}{l}\text { TNF- } \alpha \\
(2 a z 5)\end{array}$ & $\begin{array}{l}\text { VEGF } \\
(5 \mathrm{e} \times 3)\end{array}$ & $\begin{array}{l}\text { STATI } \\
\text { (3wzd) }\end{array}$ & IL-6 ( Ialu) & $\begin{array}{l}\text { PTGS2 } \\
\text { (2oyu) }\end{array}$ & $\begin{array}{l}\text { IL-2 } \\
(\operatorname{Im} 48)\end{array}$ & $\begin{array}{l}\text { NF-kB } \\
(5 \mathrm{t} 80)\end{array}$ & AKT (2uzt) & $\begin{array}{l}\text { PI3K } \\
\text { (4PS3) }\end{array}$ & IL-8 (2×6g) \\
\hline MOL000087 & \multicolumn{2}{|c|}{$\beta$-sitosterol-3-O- $\beta$-D-glucopyranoside } & -5.8 & -5.1 & -8.9 & 32.5 & -8.4 & -6.3 & -1.7 & -9.7 & -7.2 & -6.3 \\
\hline MOL000357 & \multicolumn{2}{|c|}{ eleutheroside $A$} & -6.2 & -5.3 & -8.9 & 32.2 & -8.5 & -7.6 & -2.4 & -6.1 & -7.7 & -7.3 \\
\hline MOL000358 & \multicolumn{2}{|l|}{$\beta$-sitosterol } & -6.3 & -8.1 & -9.5 & 32.4 & -8.4 & -6.3 & -6.7 & -9.5 & -6.7 & -7.6 \\
\hline MOL000392 & \multicolumn{2}{|l|}{ Formononetin } & -5.6 & -9.2 & -8.9 & 41.9 & -8.5 & -7.2 & -7.6 & -7.9 & -7.9 & -8.1 \\
\hline MOL0004I7 & \multicolumn{2}{|l|}{ Calycosin } & -5.4 & -9.1 & -9.3 & 42.1 & -8.7 & -7.3 & -7.8 & -8.2 & -8.1 & -5.1 \\
\hline MOL000469 & \multicolumn{2}{|l|}{ 7-oxo- $\beta$-sitosterol } & -6.3 & -8.4 & -9.7 & 28.5 & -7.9 & -6.2 & -6.3 & -9.2 & -7.5 & -5.3 \\
\hline MOL000497 & \multicolumn{2}{|l|}{ Licochalcone A } & -6.2 & -9.0 & -9.6 & 43.9 & -8.8 & -6.3 & -7.3 & -8.6 & -7.7 & -8.1 \\
\hline MOL000502 & \multicolumn{2}{|l|}{ Cajanin } & -5.5 & -10.4 & -8.8 & 45.3 & -8.8 & -7.1 & -7.6 & -8.1 & -7.3 & -7.8 \\
\hline MOL002565 & \multicolumn{2}{|l|}{ Medicarpin } & -5.4 & -9.5 & -9.2 & 37.6 & -9.1 & -6.8 & -8.1 & -8.6 & -7.4 & -6.3 \\
\hline MOL002663 & \multicolumn{2}{|l|}{ Skimmianine } & -5.1 & -8.0 & -8.3 & 38.7 & -8 & -5.3 & -7.2 & -7.9 & -7.3 & -7.3 \\
\hline MOL005658 & \multicolumn{2}{|l|}{ Periplogenin } & -6.3 & -5.7 & -8 & 35.3 & -7 & -5.7 & -4.6 & -8.1 & -6.0 & -6.3 \\
\hline MOL006504 & \multicolumn{2}{|l|}{$(-)$-catechin gallate } & -6.5 & -9.9 & -9.3 & 42.0 & -9.9 & -6.4 & -7.9 & -10.2 & -8.6 & -7.2 \\
\hline MOL00080I & \multicolumn{2}{|l|}{$\beta$-amyrin } & -6.7 & -1.6 & -8.7 & 19.0 & -7.7 & -6.8 & 4.8 & -5.1 & -6.1 & -8.0 \\
\hline & \multicolumn{2}{|l|}{ Original ligand } & -6.7 & -9.9 & -10.1 & $\mathrm{n} / \mathrm{a}$ & -12.7 & -7.4 & -9.7 & -9.8 & -8.9 & -9.9 \\
\hline & \multicolumn{2}{|l|}{ Average } & -5.9 & -7.6 & -9.0 & 36.2 & -8.4 & -6.5 & -5.4 & -8.2 & -7.3 & -7.0 \\
\hline
\end{tabular}

Notes: IL-6 has no crystal structure and GOLD was used for docking. A larger fitness value indicates better binding. The rest molecules were docked using AutoDock-vina software. The lower the score, the better the binding. 


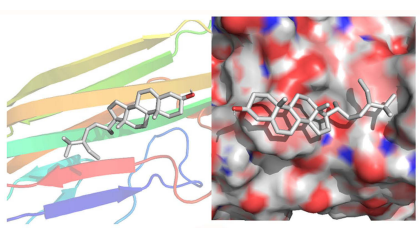

A1

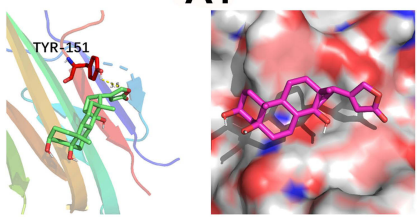

A4

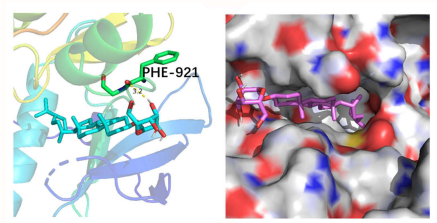

C1

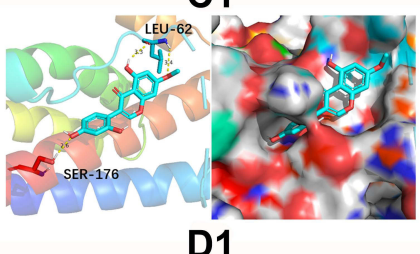

D1

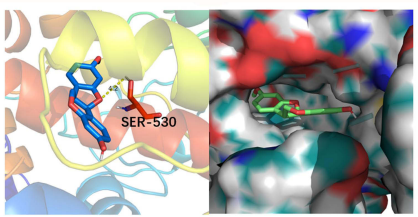

E2

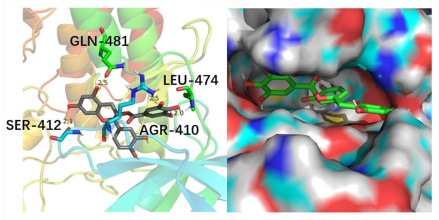

G1

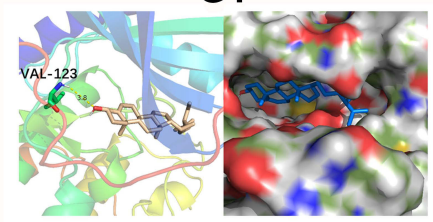

H2

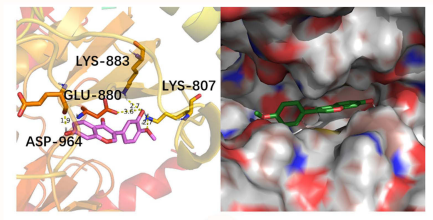

12

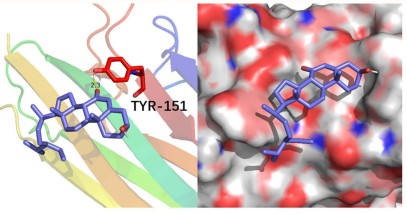

A2

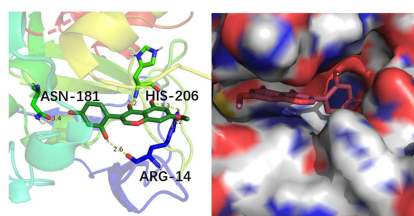

B1

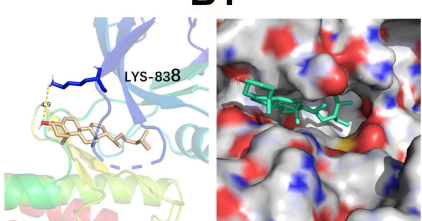

C2

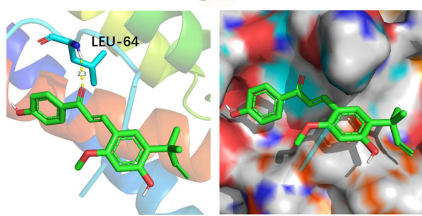

D2

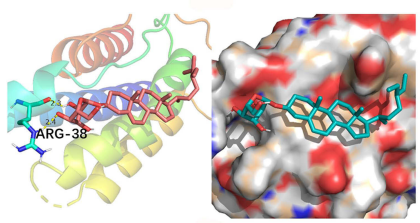

F1

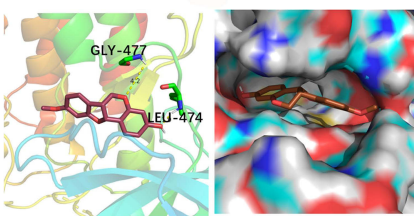

G2

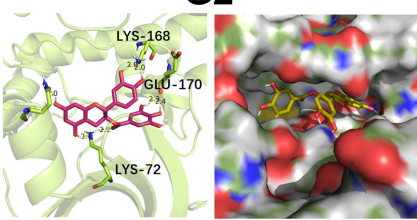

H3

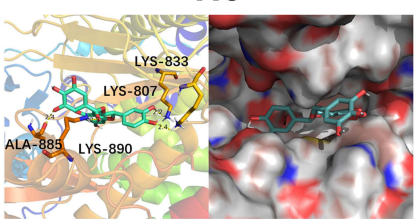

13

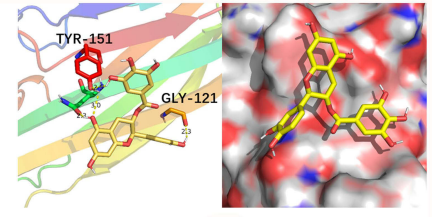

A3
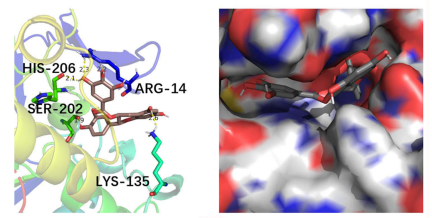

B2

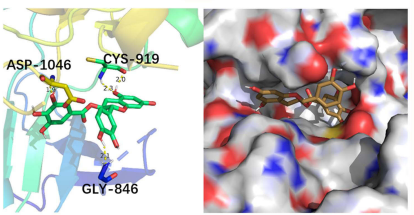

C3

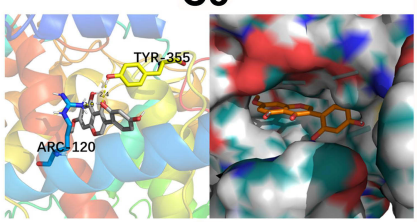

E1

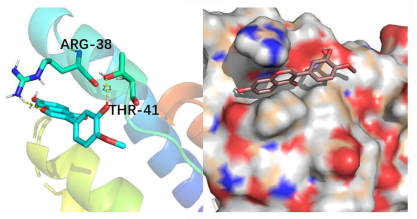

F2

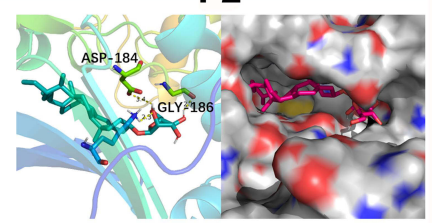

H1

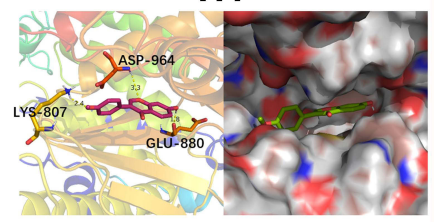

I1

Figure II Docking results of the main active compounds of SX and the key RA-associated targets. (AI) TNF- $\alpha$ and $\beta$-sitosterol; (A2) TNF- $\alpha$ and 7-oxo- $\beta$-sitosterol; (A3) TNF- $\alpha$ and (-)-catechin gallate; (A4) TNF- $\alpha$ and periplogenin. (BI) VEGF and cajanin; (B2) VEGF and (-)-catechin gallate. (CI) STATI and eleutheroside A; (C2) STATI and $\beta$-sitosterol; (C3) STATI and (-)-catechin gallate. (DI) IL-6 and cajanin; (D2) IL-6 and licochalcone A. (EI) PTGS2 and cajanin; (E2) PTGS2 and medicarpin. (FI) IL-2 and eleutheroside A; (F2) IL-2 and calycosin. (GI) NF- $\mathrm{KB}$ and (-)-catechin gallate; (G2) NF- $\kappa B$ and medicarpin. (HI) AKT and $\beta$-sitosterol-3-O- $\beta$-D-glucopyranoside. (H2) AKT and $\beta$-sitosterol; (H3) AKT and (-)-catechin gallate. (II) PI3K and eleutheroside A;(I2) PI3K and $\beta$-sitosterol; (I3) PI3K and (-)-catechin gallate. 
Table 6 Effect of SX on Joint Swelling in CIA Rats (Mean \pm SEM, $n=10$ )

\begin{tabular}{|c|c|c|c|c|c|}
\hline Group & 7d & $14 \mathrm{~d}$ & $23 \mathrm{~d}$ & $30 \mathrm{~d}$ & $37 \mathrm{~d}$ \\
\hline Nor group & $1.199 \pm 0.060$ & $1.241 \pm 0.082$ & $1.306 \pm 0.067$ & $1.341 \pm 0.063$ & $1.400 \pm 0.092$ \\
\hline Mod group & $1.17 \mid \pm 0.075$ & $1.616 \pm 0.085^{* * *}$ & $1.621 \pm 0.05 I^{* * *}$ & $1.645 \pm 0.073^{* * *}$ & $1.666 \pm 0.077^{* *}$ \\
\hline GTW group & $1.216 \pm 0.074$ & $1.549 \pm 0.070^{* * *}$ & $1.404 \pm 0.080 * * \# \#$ & $1.353 \pm 0.069^{\# \# \#}$ & $1.390 \pm 0.085^{\# \# \#}$ \\
\hline $\mathrm{SX} 40 \mathrm{~g} / \mathrm{kg}$ group & $1.169 \pm 0.049$ & $1.554 \pm 0.088^{* * *}$ & $1.432 \pm 0.070 * * \# \#$ & $1.345 \pm 0.065^{\# \# \#}$ & $1.416 \pm 0.200^{\# \#}$ \\
\hline $\mathrm{SX} 20 \mathrm{~g} / \mathrm{kg}$ group & $1.203 \pm 0.057$ & $1.600 \pm 0.029 * * *$ & $1.543 \pm 0.064^{* * * \#}$ & $1.484 \pm 0.034 * * * \# \#$ & $1.419 \pm 0.102^{\# \Delta \Delta \Delta}$ \\
\hline $\mathrm{SX} 10 \mathrm{~g} / \mathrm{kg}$ group & I. $178 \pm 0.062$ & $1.590 \pm 0.060^{* * *}$ & $1.578 \pm\left. 0.09\right|^{* * *} \triangle \Delta$ & 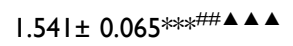 & $1.519 \pm 0.236^{\# \Delta \Delta \Delta}$ \\
\hline
\end{tabular}

Notes: I4d: *Comparison with Nor group, ***P<0.00I (Mod vs Nor, ***P=0.000; GTW vs Nor, *** ${ }^{2}=0.000 ; \mathrm{SX} 40 \mathrm{~g} / \mathrm{kg}$ vs Nor, $* * * P=0.000 ; \mathrm{SX} 20 \mathrm{~g} / \mathrm{kg}$ vs Nor, $* * * P=0.000$; $\mathrm{SX} 10 \mathrm{~g} / \mathrm{kg}$ vs Nor, ${ }^{* * * P=0.000) ; ~ 23 d: ~}{ }^{*}$ Comparison with Nor group, ${ }^{* *} P<0.0 \mathrm{I}, * * P<0.00 \mathrm{I}$ (Mod vs Nor, $* * * P=0.000 ; \mathrm{GTW}$ vs Nor, $* * P=0.009 ; \mathrm{SX} 40 \mathrm{~g} / \mathrm{kg}$ vs Nor, $* * P=0.00 \mathrm{I}$;

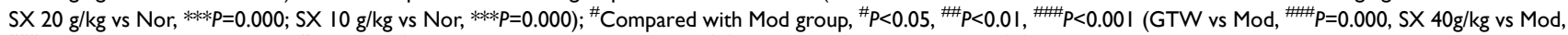

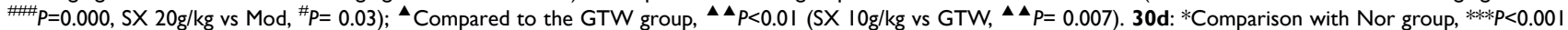

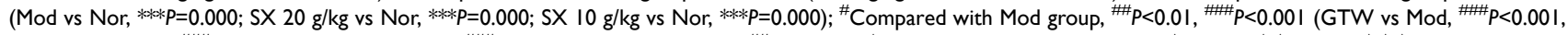

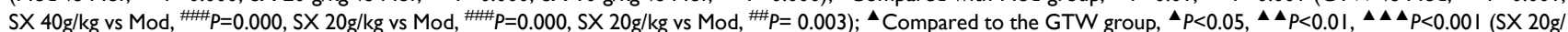

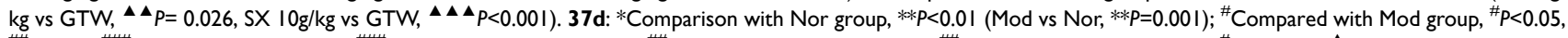
${ }^{\#} P<0.01,{ }^{\#} P<0.00$ I (GTW vs Mod, ${ }^{\# \#} P=0.000, \mathrm{SX} 40 \mathrm{~g} / \mathrm{kg}$ vs Mod, ${ }^{\#} P=0.0014, \mathrm{SX} 20 \mathrm{~g} / \mathrm{kg}$ vs Mod, ${ }^{\#} P=0.002$, SX 20g/kg vs Mod, ${ }^{\#} P=0.049$ ); ${ }^{\wedge}$ Compared to the GTW

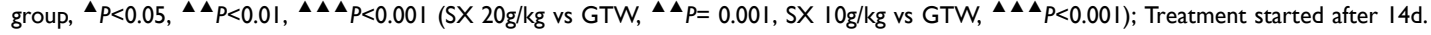

Abbreviations: SX, Sidaxue; CIA, collagen-induced arthritis; GTW, Tripterygium wilfordii polyglycoside tablet.

hind limbs in each treatment group gradually decreased after drug administration. According to the results of the degree of foot swelling (Table 6, Figure 12A) and AI score (Table 7, Figure 12B), the swelling of the knees, ankles and interdigital joints of the hind limbs of the rats in each administration group gradually decreased after the administration. After 2 weeks of treatment, compared with the Mod group, the SX $40 \mathrm{~g} / \mathrm{kg}$, SX $20 \mathrm{~g} / \mathrm{kg}$ and SX $10 \mathrm{~g} / \mathrm{kg}$ and GTW groups showed decreased foot swelling $(P=0.000, P=0.003, P=0.000, P=0.000, P<0.001, P<$ 0.01). After 3 weeks, all the SX groups and the GTW group showed decreased foot swelling, compared to the Mod group, with the greatest decrease in the SX $40 \mathrm{~g} / \mathrm{kg}$ and GTW groups $(P=0.0014, P=0.000, P<0.001)$ (Table 6). After 2 weeks of treatment (30 days after the beginning of CIA induction), the AI score of SX $40 \mathrm{~g} / \mathrm{kg}$ and GTW groups were lower than that of the Mod group $(P=0.018, P=0.003, P<0.05, P<0.01)$. This beneficial effect was

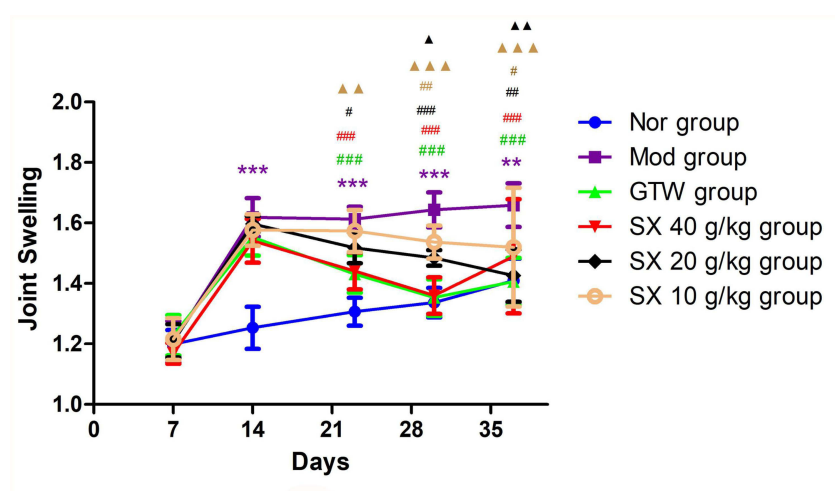

A

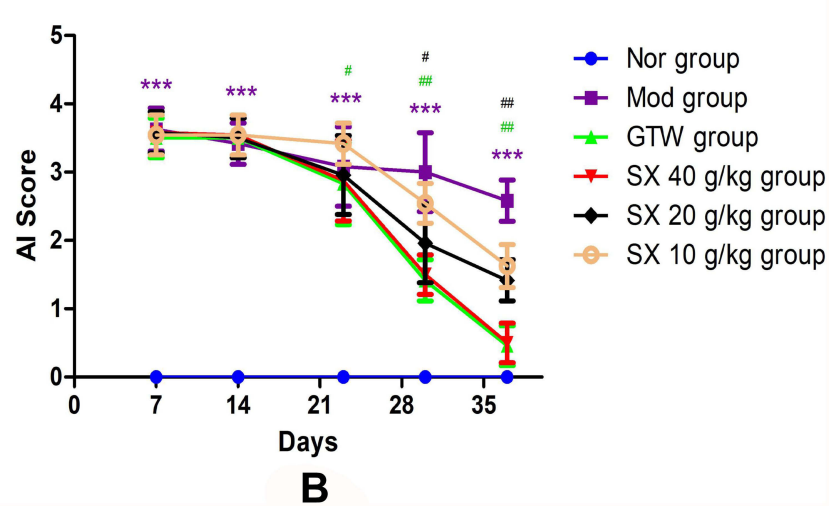

Figure 12 (A) The degree of foot swelling of CIA rat. 14d: *Comparison with Nor group, $* * * P<0.001$ (Mod vs Nor, $* * * P=0.000$; GTW vs Nor, $* * * P=0.000$; SX 40 g/kg vs Nor, $* * * P=0.000 ; \mathrm{SX} 20 \mathrm{~g} / \mathrm{kg}$ vs Nor, $* * * P=0.000 ; \mathrm{SX} 10 \mathrm{~g} / \mathrm{kg}$ vs Nor, $* * * P=0.000$ ); 23d: $*$ Comparison with Nor group, $* * P<0.0 \mathrm{I}$, $* * P<0.00 \mathrm{I}$ (Mod vs Nor, $* * * P=0.000 ;$ GTW vs Nor, ${ }^{* * P} P=0.009 ; \mathrm{S} X 40 \mathrm{~g} / \mathrm{kg}$ vs Nor, ${ }^{* * P} P=0.00 \mathrm{I} ; \mathrm{S} X 20 \mathrm{~g} / \mathrm{kg}$ vs Nor, ${ }^{* * *} P=0.000 ; \mathrm{S} \times 10 \mathrm{~g} / \mathrm{kg}$ vs Nor, $\left.{ }^{* * *} P=0.000\right) ;{ }^{\#}$ Compared with Mod group, ${ }^{\#} P<0.05,{ }^{\#} P<0.01,{ }^{\# \#} P<0.001$

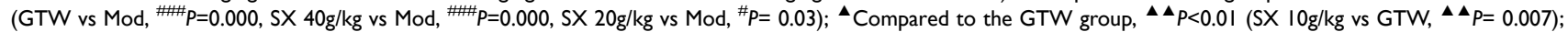
30d: *Comparison with Nor group, ${ }^{*} * *<<0.001$ (Mod vs Nor, $* * * P=0.000 ; \mathrm{SX} 20 \mathrm{~g} / \mathrm{kg}$ vs Nor, *** $P=0.000$; SX $10 \mathrm{~g} / \mathrm{kg}$ vs Nor, $* * * P=0.000$ ); ${ }^{\#}$ Compared with Mod group,

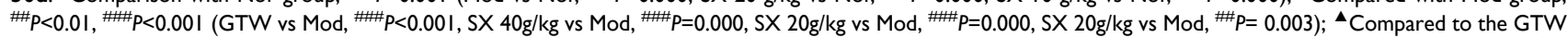

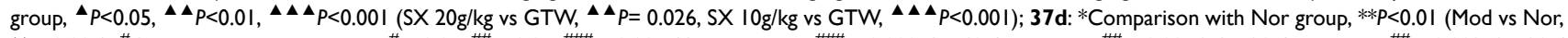
**P=0.00I); ${ }^{\#}$ Compared with Mod group, ${ }^{\# P<0.05, ~}{ }^{\# P} P<0.01,{ }^{\#} P<0.001$ (GTW vs Mod, ${ }^{\# \# P=0.000, S X ~ 40 g / k g ~ v s ~ M o d, ~}{ }^{\#} P=0.0014, S X 20 \mathrm{~g} / \mathrm{kg}$ vs Mod, ${ }^{\#} P=0.002, \mathrm{SX} 20 \mathrm{~g} /$

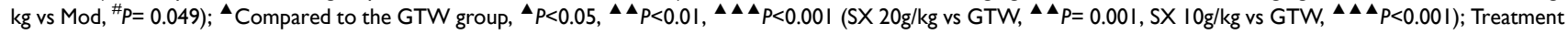
started after 14d. (B) The Al score of CIA rats. 7d: $*$ Comparison with Nor group, $* * * P<0.00$ I; I4d: $*$ Comparison with Nor group, $* * P=0.005$; 23d: $*$ Comparison with Nor group, ${ }^{* * *} P<0.00$ I; ${ }^{\#}$ Compared with Mod group, ${ }^{\#} P<0.05$ (GTW vs Mod, ${ }^{\#} P=0.049$ ); 30d: *Comparison with Nor group, ${ }^{* * * P<0.00 I ;}{ }^{\#}$ Compared with Mod group, ${ }^{\#} P<0.05$,

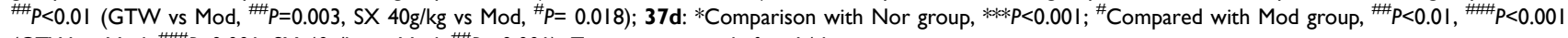
(GTW vs Mod, ${ }^{\# \#} P<0.001$, SX 40g/kg vs Mod, ${ }^{\#} P=0.001$ ); Treatment started after $14 \mathrm{~d}$.

Abbreviations: SX, Sidaxue; CIA, collagen-induced arthritis; GTW, Tripterygium wilfordii polyglycoside tablet; Al, arthritis index. 
Table 7 Effect of SX on the AI Score of CIA Rats (Mean \pm SEM, $n=10$ )

\begin{tabular}{|l|l|l|l|l|l|}
\hline Group & $\mathbf{7 ~ d}$ & 14 d & 23 d & 30 d & 37 d \\
\hline Nor group & $0.00 \pm 0.00$ & $0.00 \pm 0.00$ & $0.00 \pm 0.00$ & $0.00 \pm 0.00$ & $0.00 \pm 0.00$ \\
Mod group & $3.875 \pm 0.354^{* * *}$ & $3.500 \pm 0.535^{* *}$ & $3.750 \pm 0.463^{* * *}$ & $3.500 \pm 0.535^{* * *}$ & $3.625 \pm 0.518^{* * *}$ \\
GTW group & $3.500 \pm 0.535$ & $3.500 \pm 0.535$ & $2.500 \pm 0.535^{\#}$ & $1.250 \pm 0.463^{\# \#}$ & $0.375 \pm 0.518^{\# \#}$ \\
SX 40 g/kg group & $3.750 \pm 0.463$ & $3.625 \pm 0.518$ & $2.625 \pm 0.518$ & $1.500 \pm 0.535^{\#}$ & $0.500 \pm 0.535^{\# \#}$ \\
SX 20 g/kg group & $3.750 \pm 0.463$ & $3.500 \pm 0.535$ & $2.875 \pm 0.641$ & $1.875 \pm 0.641$ & $1.250 \pm 0.463$ \\
SX 10 g/kg group & $3.083 \pm 1.456$ & $3.625 \pm 0.518$ & $3.250 \pm 0.623$ & $2.625 \pm 0.518$ & $1.875 \pm 0.641$ \\
\hline
\end{tabular}

Notes: 7d: *Comparison with Nor group, $* * * P<0.00$ I; I4d: *Comparison with Nor group, $* * P=0.005$; 23d: *Comparison with Nor group, $* * * P<0.001$; ${ }^{*}$ Compared with Mod group, ${ }^{\#} P<0.05$ (GTW vs Mod, ${ }^{\#} P=0.049$ ); 30d: ${ }^{*}$ Comparison with Nor group, ${ }^{* * *} P<0.001$; ${ }^{\#}$ Compared with Mod group, ${ }^{\#} P<0.05,{ }^{\#} P<0.01$ (GTW vs Mod, ${ }^{\#} P=0.003$,

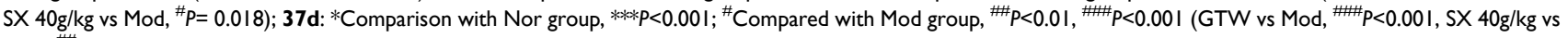
Mod, $\left.{ }^{\#} P=0.001\right)$; Treatment started after 14d.

Abbreviations: SX, Sidaxue; AI, arthritis index; CIA, collagen-induced arthritis; GTW, Tripterygium wilfordii polyglycoside tablet.

even more striking after 3 weeks of drug administration (37 days after CIA induction) $(P=0.001, P<0.01$; Table 7). (See Supplementary Table S7 for median data).

\section{Histological Analysis of Rat Synovial Tissues}

It can be seen from the HE slices that the black arrows represent the joint cavity and the blue arrows represent the blood vessels. The pictures are all magnified 100 times. There were no obvious inflammatory cell infiltrating into the toe synovial tissues of the Nor group (Figure 13A). In the Mod group, a large number of inflammatory cells, fibrous tissue hyperplasia and edema were seen in the dermal papillary layers and reticular layers in the joints of CIA rats. Occasionally, the tissue structure of the different layers was disordered and the epithelial tissue was destroyed (Figure 13B). In the GTW group, the inflammatory cell number and infiltration were significantly reduced (Figure 13C). A very small amount of scattered inflammatory cells was occasionally noticed in tissues from the SX 40 $\mathrm{g} / \mathrm{kg}$ group (Figure 13D). In the SX $20 \mathrm{~g} / \mathrm{kg}$ group, the inflammatory cell infiltration, the fibrous tissue proliferation and the edema were all reduced (Figure 13E). In contrast, joints from the SX $10 \mathrm{~g} / \mathrm{kg}$ group still showed more inflammatory cell infiltration and tissue edema than that of the Nor group (Figure 13F).

\section{Effect of SX on Serum IL-2, TNF- $\alpha$, VEGF-A, IL-6 Level}

The content of IL-2 and TNF- $\alpha$ in the serum of rats was then changed. Compared with the Nor group, the Mod group increased the content (IL-2: $P=0.0215, P<0.05$;TNF- $\alpha$ : $P=0.0064, P<0.01$ ); the GTW group, SX $40 \mathrm{~g} / \mathrm{kg}$ and SX $20 \mathrm{~g} / \mathrm{kg}$ were all lower than the Mod group (IL-2: $P=0.0324,0.0412,0.026, P<0.05$; TNF- $\alpha$ : $P=0.0346,0.0275,0.0167, P<0.05$ ); The content of the SX $10 \mathrm{~g} / \mathrm{kg}$ group was higher than that of the GTW group (IL-2: $P=0.0381, P<0.05$;TNF- $\alpha$ : $P=0.0219$; $P<0.05)$, and the rest of the SX group was not statistically significant compared with the GTW group $(P>0.05)$ (Table 8 , Figure $14 \mathrm{~A}$ and $\mathrm{B})$.

The content of VEGF-A and IL-6 in the serum of rats was next changed. Compared with the Nor group, the Mod group was significantly higher (VEGF-A: $P=0.0024, P<0.01$; IL-6: $P=0.0078, P<0.01$ ); the GTW treatment groups, SX $40 \mathrm{~g} / \mathrm{kg}$ and $20 \mathrm{~g} / \mathrm{kg}$ were all lower than the Mod group (VEGF-A: $P=0.0062,0.0039,0.0273 ; P<0.01$ or $P<0.05 ; \mathrm{IL}-6$ : $P=0.0043,0.0059, P=0.0357, P<0.01$ or $P<0.05)$; the SX $40 \mathrm{~g} / \mathrm{kg}$ group and SX $20 \mathrm{~g} / \mathrm{kg}$ group when compared with the GTW group is not statistically significant $(P>0.05)$; the $\mathrm{SX} 10 \mathrm{~g} / \mathrm{kg}$ group is higher than the GTW group (VEGF-A: $P=0.0318, P<0.05$; IL-6: $P=0.0371, P<0.05$ ). Thus, serum VEGF-A and IL-6 level did not show linear relationship with SX doses (Table 8, Figure 14C and D).

The ELISA results show that SX can reduce the content of IL-2, TNF- $\alpha$, VEGF-A, and IL-6, thereby reducing the synovial inflammation and pannus, achieving therapeutic effects, and predicting the target analysis of SX treatment of RA by network pharmacology. 

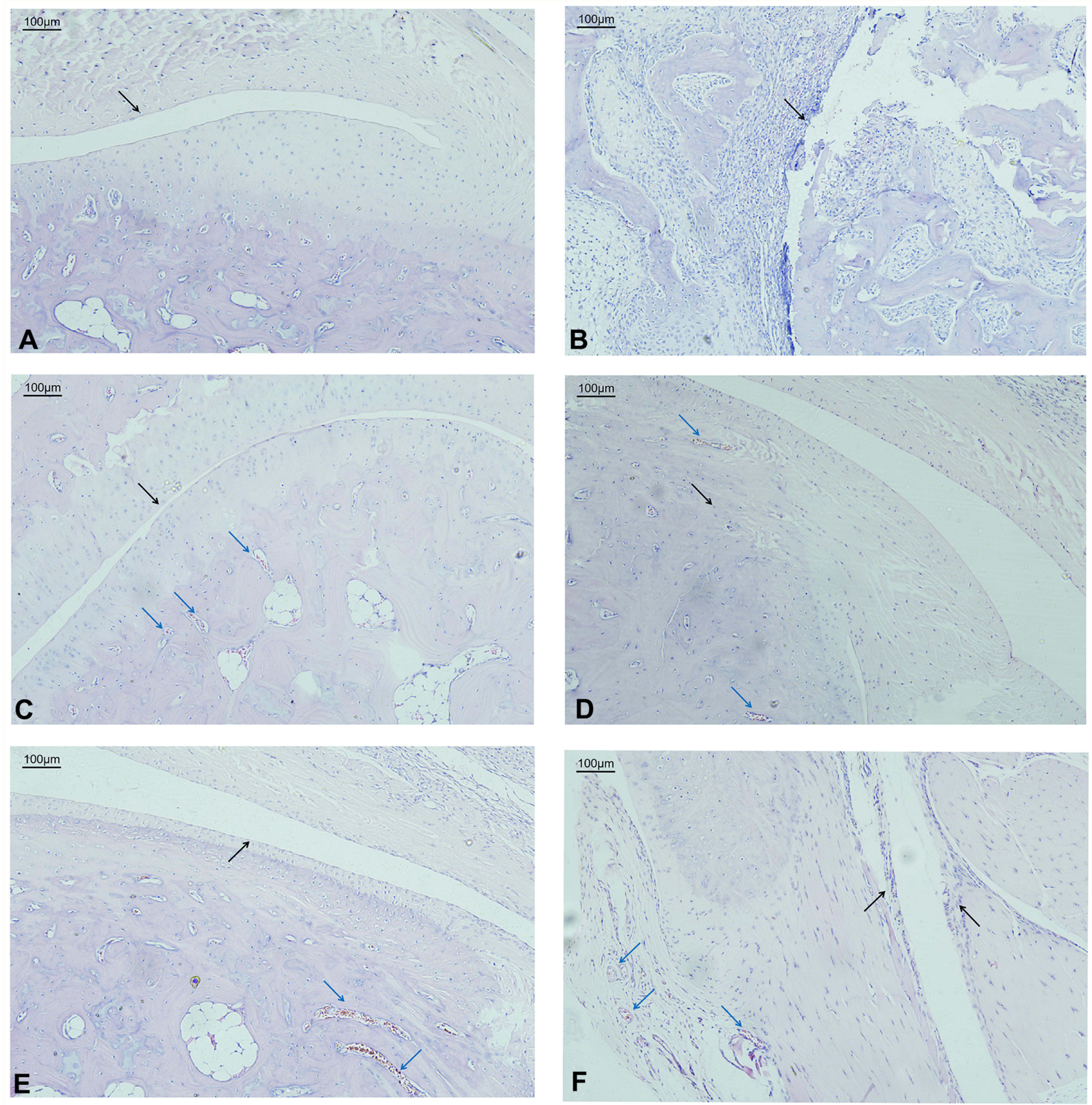

Figure 13 Histology of healthy and CIA rat synovium. Representative pictures of joint sections from non-induced (A) or CIA-induced rats (B-F), non-treated (A and B) or treated with GTW (C) or SX (D-F). The sections were stained by H\&E (I00X). (A), Nor group; (B), Mod group; (C), GTW group; (D), SX 40 g/kg group; (E), SX 20 g/kg group; (F), SX $10 \mathrm{~g} / \mathrm{kg}$ group. Magnification: $100 \times$.

Notes: Black arrows show synovial inflammatory cell infiltrates and hyperplasia; red arrows show newly formed blood vessels.

\section{Effect of SX on NF-кBp65, STATI, PTGS2, PI3K, AKT and VEGF-A mRNA Level in Synovial Tissues of CIA Rats}

After 21 days of administration, the expression of NF- $\mathrm{kB}$ p65 mRNA increased compared with the Nor group, and the content of the Mod group increased $(P=0.0064, P<0.01)$; compared with the Mod group, the content of the SX $40 \mathrm{~g} / \mathrm{kg}$ group, the SX 20g/kg group and the GTW group decreased ( $P=0.026,0.023, P=0.0076, P<0.05, P<0.01)$; There was no statistically significant difference between the SX $10 \mathrm{~g} / \mathrm{kg}$ and Mod groups $(P>0.05)$; the SX $10 \mathrm{~g} / \mathrm{kg}$ increased in content 
Table 8 Serum Cytokine Levels in CIA Rats (Mean \pm SEM, $n=10$ )

\begin{tabular}{|l|l|l|l|l|}
\hline Group & $\begin{array}{l}\text { IL-2 Level } \\
(\mathbf{p g} / \mathbf{m L})\end{array}$ & $\begin{array}{l}\text { TNF- } \alpha \text { Level } \\
\mathbf{( p g / m L )}\end{array}$ & $\begin{array}{l}\text { VEGF-A Level } \\
\mathbf{( p g / m L )}\end{array}$ & $\begin{array}{l}\text { IL-6 Level } \\
\mathbf{( p g / m L})\end{array}$ \\
\hline Nor group & $128.63 \pm 11.53$ & $29.79 \pm 4.62$ & $50.55 \pm 8.62$ & $31.84 \pm 5.67$ \\
Mod group & $178.25 \pm 12.31^{* \Delta}$ & $61.84 \pm 6.76^{* * \Delta}$ & $159.71 \pm 5.69^{* * \Delta}$ & $78.68 \pm 7.31^{* * \Delta}$ \\
GTW group & $131.36 \pm 13.28^{\#}$ & $38.84 \pm 6.54^{\#}$ & $64.92 \pm 5.60^{\# \#}$ & $41.23 \pm 8.13^{\# \#}$ \\
SX 40 g/kg group & $137.12 \pm 12.42^{\#}$ & $31.63 \pm 5.31^{\#}$ & $69.64 \pm 8.26^{\# \#}$ & $32.15 \pm 5.63^{\# \#}$ \\
SX 20 g/kg group & $154.32 \pm 13.25^{\#}$ & $40.23 \pm 6.13^{\#}$ & $88.92 \pm 10.83^{\#}$ & $39.16 \pm 5.89^{\#}$ \\
SX 10 g/kg group & $175.61 \pm 11.78^{\Delta}$ & $55.34 \pm 4.67^{\Delta}$ & $130.05 \pm 12.20^{\Delta}$ & $56.31 \pm 5.43^{\Delta}$ \\
\hline
\end{tabular}

Notes: Compared to the Nor group, (IL-2: *P=0.02I5; TNF- $\alpha: * * P=0.0064$; VEGF-A: **P=0.0024; IL-6: **P=0.0078); Compared to the Mod group, (IL2: ${ }^{\#} P=0.0324,0.0412,0.026$; TNF- $\alpha:{ }^{\#} P=0.0346,0.0275,0.0167$; VEGF-A: ${ }^{\#} P=0.0062,0.0039,{ }^{\#} P=0.0273 ;$ IL-6: ${ }^{\# \#} P=0.0043,0.0059,{ }^{\#} P=0.0357$ );

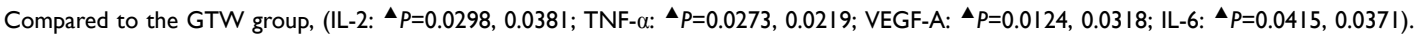

Abbreviations: CIA, collagen-induced arthritis; GTW, Tripterygium wilfordii polyglycoside tablet; SX, Sidaxue.

compared with the GTW group $(P=0.0072, P<0.01)$, SX $40 \mathrm{~g} / \mathrm{kg}$ group, the SX $20 \mathrm{~g} / \mathrm{kg}$ group and the GTW group exhibited no significant difference $(P>0.05)$ (Figure 15A).

On comparison with the Nor group, the STAT1 mRNA content in the Mod group increased $(P=0.0018, P<0.01)$; Compared with the Mod group, the STAT1 mRNA content in the SX $40 \mathrm{~g} / \mathrm{kg}$ group, the SX $20 \mathrm{~g} / \mathrm{kg}$ group and the GTW group decreased $(P=0.0085,0.0038, P=0.031, P<0.05, P<0.01)$. Among them, SX 40g $/ \mathrm{kg}$ can significantly downregulate the expression of STAT1 mRNA $(P<0.01)$. Compared with the GTW group, the content of the SX $20 \mathrm{~g} / \mathrm{kg}$ group and the SX 10g/kg group increased $(P=0.041, P=0.0068, P<0.05, P<0.01)$; compared with the GTW group, the SX $40 \mathrm{~g} / \mathrm{kg}$ group revealed little difference $(P>0.05)$ (Figure 15B).

Compared with the Nor group, the PTGS2 mRNA expression level increased in the Mod group $(P=0.0081, P<0.01)$; compared with the Mod group, the content in the SX 40g/kg group and the GTW group decreased $(P=0.036, P=0.0067$, $P<0.05, P<0.01)$; SX $20 \mathrm{~g} / \mathrm{kg}$ There was no statistically significant difference between the $\mathrm{SX} 10 \mathrm{~g} / \mathrm{kg}$ and the Mod groups $(P>0.05)$; the SX $20 \mathrm{~g} / \mathrm{kg}$ group and the SX $10 \mathrm{~g} / \mathrm{kg}$ group were higher than the GTW group $(P=0.023, P=0.0037, P<0.05$, $P<0.01)$. There was little difference between the SX $40 \mathrm{~g} / \mathrm{kg}$ group and the GTW group $(P>0.05)$ (Figure 15C).

Compared with the Nor group, the expression of PI3K mRNA in the Mod group increased $(P=0.0074, P<0.01)$; compared with the Mod group, the expression of the SX $40 \mathrm{~g} / \mathrm{kg}$ group, the SX $20 \mathrm{~g} / \mathrm{kg}$ group and the GTW group decreased $(P=0.024, P=0.0088,0.0049, P<0.05, P<0.01)$. There was no significant difference between the SX $10 \mathrm{~g} / \mathrm{kg}$ and the Mod groups $(P>0.05)$; the expression level of the SX $10 \mathrm{~g} / \mathrm{kg}$ group was higher than that of the GTW group $(P=0.031, P<0.05)$, and the SX $40 \mathrm{~g} / \mathrm{kg}$ group. There was little difference between the SX $20 \mathrm{~g} / \mathrm{kg}$ group and the GTW group $(P>0.05)$ (Figure 15D).

Compared with the Nor group, the AKT mRNA content in the Mod group increased ( $P=0.0091, P<0.01$ ); Compared with the Mod group, the AKT mRNA content in the GTW group, the SX 40g/kg group and the SX $20 \mathrm{~g} / \mathrm{kg}$ group decreased $(P=0.0095,0.0056, P=0.042, P<0.01, P<0.05)$. Among them, SX 40g $/ \mathrm{kg}$ can significantly down-regulate the expression of AKT mRNA $(P<0.01)$. Compared with the GTW group, the content of the SX $20 \mathrm{~g} / \mathrm{kg}$ group and the SX $10 \mathrm{~g} / \mathrm{kg}$ group increased $(P=0.0431, P=0.0075, P<0.05, P<0.01)$; compared with the GTW group, the $\mathrm{SX} 40 \mathrm{~g} / \mathrm{kg}$ group had little difference $(P>0.05)$ (Figure 15E).

Compared with the Mod group, the VEGF-A mRNA content in the SX $40 \mathrm{~g} / \mathrm{kg}$ group, the SX $20 \mathrm{~g} / \mathrm{kg}$ group and the SX $10 \mathrm{~g} / \mathrm{kg}$ group all decreased $(P=0.0075, P=0.039,0.028, P<0.01, P<0.05)$, and the $\mathrm{SX} 40 \mathrm{~g} / \mathrm{kg}$ could significantly down-regulate the expression of VEGF-A mRNA $(P=0.0075, P<0.01)$. Compared with the GTW group, the SX $40 \mathrm{~g} / \mathrm{kg}$ and the SX $10 \mathrm{~g} / \mathrm{kg}$ group revealed little difference $(P>0.05)$; for the SX $20 \mathrm{~g} / \mathrm{kg}$ group compared with GTW, the difference was statistically significant $(P=0.019, P<0.05)$ (Figure 15F).

Network pharmacology analysis and molecular docking results suggest that NF-אBp65, STAT1, PTGS2, PI3K, AKT and VEGF-A are important targets for SX treatment of RA. Its mechanism of action is to control inflammation, reduce pannus, and achieve the effect of protecting joints. The qRT-PCR results indicate that SX can achieve the therapeutic effect of RA by reducing the gene content of NF-kBp65, STAT1, PTGS2, PI3K, AKT and VEGF-A. 
A

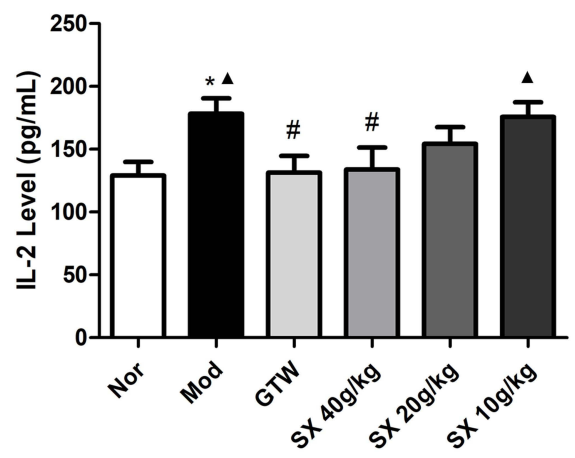

C

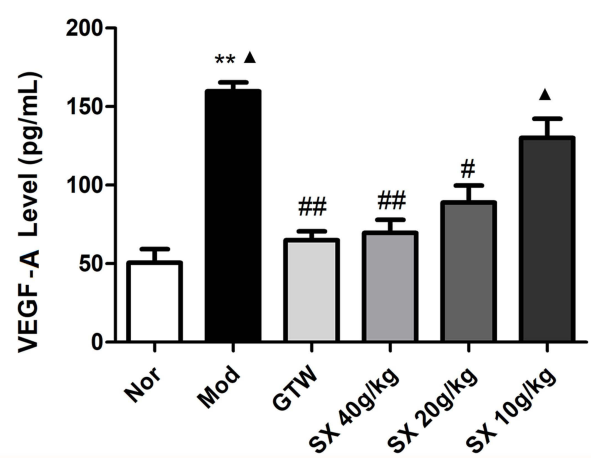

B

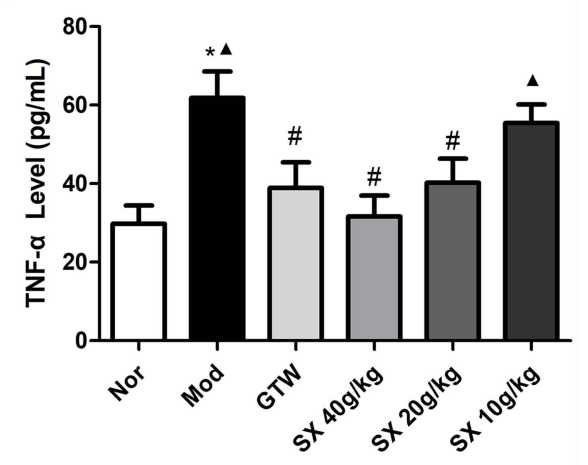

D

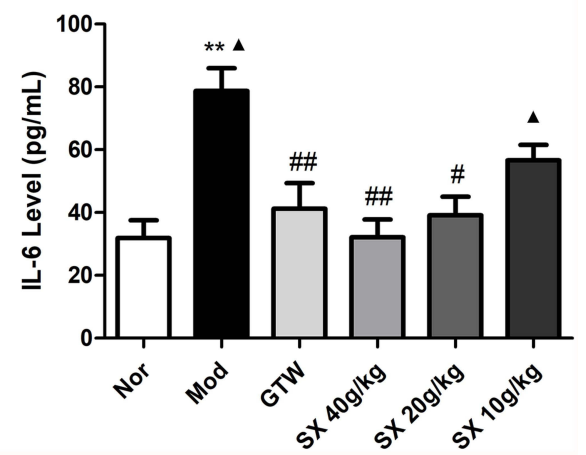

Figure 14 The effect of SX on the rat's serum levels of IL-2, TNF- $\alpha$, VEGF-A and IL-6 (Mean \pm SEM, $n=10$ ). (A) IL-2: *Compared to the Nor group, *P<0.05, (Mod group vs Nor group, $\left.{ }^{*} P=0.0215\right)$; ${ }^{\#}$ Compared to the Mod group, ${ }^{\#} P<0.05,{ }^{\# \#} P<0.01$ (GTW group vs Mod group, ${ }^{*} P=0.029 ; S X 40 \mathrm{~g} / \mathrm{kg}$ group vs Mod group, ${ }^{\#} P=0.02 \mathrm{I}$ ); ${ }^{\wedge}$ Compared to the GTW group, ${ }^{\wedge} P<0.05$ (Mod group vs GTW group, ${ }^{\wedge} P=0.0298 ; \mathrm{SX} 10 \mathrm{~g} / \mathrm{kg}$ group vs GTW group, ${ }^{\boldsymbol{\Delta}} P=0.038 \mathrm{I}$ ). (B) TNF- $\alpha:{ }^{*} \mathrm{Compared}$ to the Nor group, $* * P<0.0 \mathrm{I},($ Mod group vs Nor group, $\left.{ }^{* * P} P=0.0064\right) ;{ }^{\#}$ Compared to the Mod group, ${ }^{\#} P<0.05,{ }^{\#} P<0.01$ (GTW group vs Mod group, ${ }^{\#} P=0.0346 ; S X 40 \mathrm{~g} / \mathrm{kg}$ group vs Mod group, ${ }^{\#} P=0.0275, \mathrm{SX}$ $20 \mathrm{~g} / \mathrm{kg}$ group vs Mod group, $\left.{ }^{\#} P=0.0167\right) ;{ }^{\Delta}$ Compared to the GTW group, ${ }^{\Delta} P<0.05$ (Mod group vs GTW group, ${ }^{\Delta} P=0.0273 ;$ SX I0g/kg group vs GTW group, ${ }^{\Delta} P=0.0219$ ). (C) VEGF-A: *Compared to the Nor group, ${ }^{* * P}<0.01$, (Mod group vs Nor group, $\left.{ }^{* * P} P=0.0024\right) ;{ }^{*}$ Compared to the Mod group, ${ }^{\#} P<0.05$, ${ }^{*} P<0.01$ (GTW group vs Mod group, ${ }^{\#} P=0.0062 ; S X 40 \mathrm{~g} / \mathrm{kg}$ group vs Mod group, ${ }^{\#} P=0.0039, \mathrm{SX} 20 \mathrm{~g} / \mathrm{kg}$ group vs Mod group, ${ }^{\#} P=0.0273$ ); ${ }^{\wedge}$ Compared to the GTW group, ${ }^{\wedge} P<0.05$ (Mod group vs GTW group,

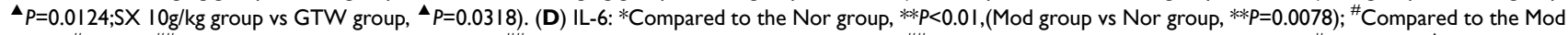
group, ${ }^{\#} P<0.05,{ }^{\# P} P<0.01$ (GTW group vs Mod group, ${ }^{\# P} P=0.0043 ; S X 40 \mathrm{~g} / \mathrm{kg}$ group vs Mod group, ${ }^{\#} P=0.0059, \mathrm{SX} 20 \mathrm{~g} / \mathrm{kg}$ group vs Mod group, ${ }^{\#} P=0.0357$ ); ${ }^{\wedge}$ Compared to the GTW group, ${ }^{\boldsymbol{\Delta}} P<0.05$ (Mod group vs GTW group, ${ }^{\boldsymbol{\Delta}} P=0.04 \mathrm{I}$; $\mathrm{SX}$ 10g/kg group vs GTW group, ${ }^{\boldsymbol{\Delta}} P=0.037 \mathrm{I}$ ).

Abbreviations: CIA, collagen-induced arthritis; GTW, Tripterygium wilfordii polyglycoside tablet; SX, Sidaxue.

In addition, the Fold change of NF- $\kappa$ Bp65, STAT1, PTGS2, PI3K, AKT and VEGF-A mRNA expression showed that compared with the Nor group, the Mod group had different changes, ${ }^{*} P<0.05$, ${ }^{*} P<0.01$; Compared with the Mod group, the GTW group and the SX $40 \mathrm{~g} / \mathrm{kg}$ group have different changes, ${ }^{\#} P<0.05,{ }^{\#} P<0.01$; Compared with the GTW group, the Mod group and the SX $10 \mathrm{~g} / \mathrm{kg}$ group have different changes, ${ }^{\mathbf{\Delta}} P<0.05,{ }^{\boldsymbol{\Delta}} P<0.01$. (The Fold change of gene expression is shown in Supplementary Table S8).

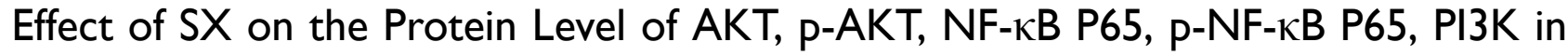 Synovial Tissues of CIA Rats}

After administration for 21 days, Western Blot detected the protein expression of the CIA rat synovial cells AKT, p-AKT,

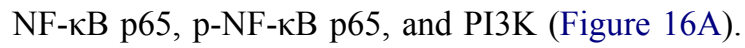

AKT total protein expression: Compared with the Nor group, the Mod group was significantly up-regulated $(P=0.0015, P<0.01)$; Compared with the Mod group, the SX $40 \mathrm{~g} / \mathrm{kg}$ group, SX $20 \mathrm{~g} / \mathrm{kg}$ group, the GTW group was significantly down-regulated $(P=0.0061, P=0.021, P=0.029, P<0.05)$. There was no significant difference between the SX $10 \mathrm{~g} / \mathrm{kg}$ group and the Mod group $(P>0.05)$, while the difference between the SX $10 \mathrm{~g} / \mathrm{kg}$ group and the GTW group was statistically significant $(P=0.0027, P<0.01)$ (Figure 16B). 
A

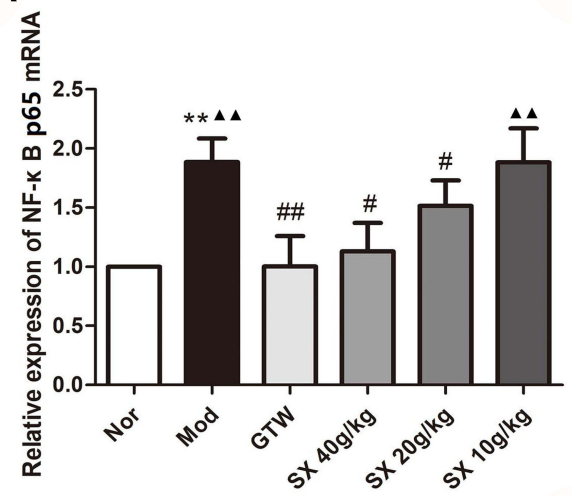

C

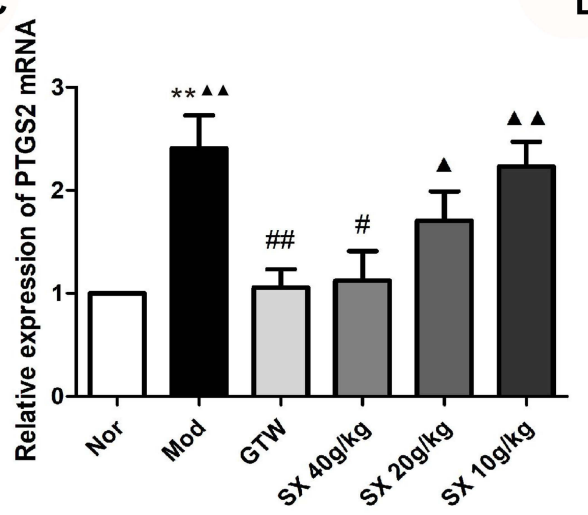

E

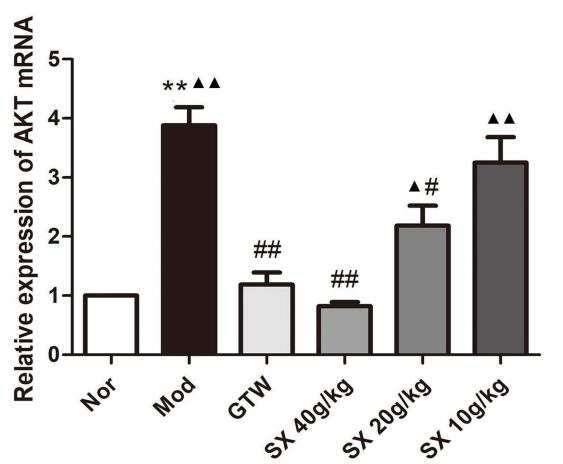

B

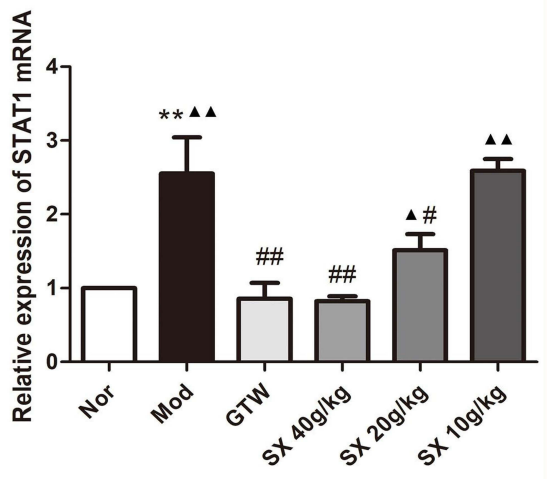

D

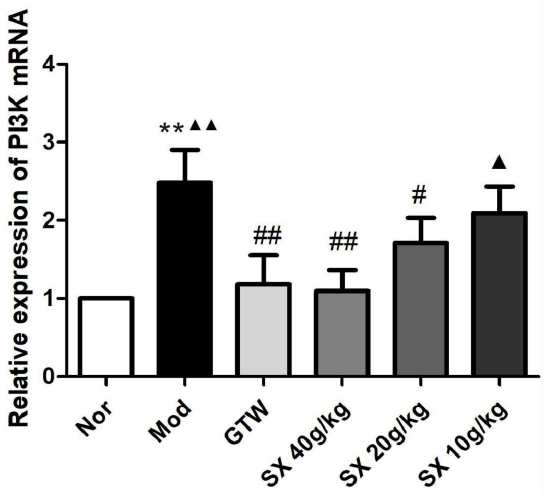

F

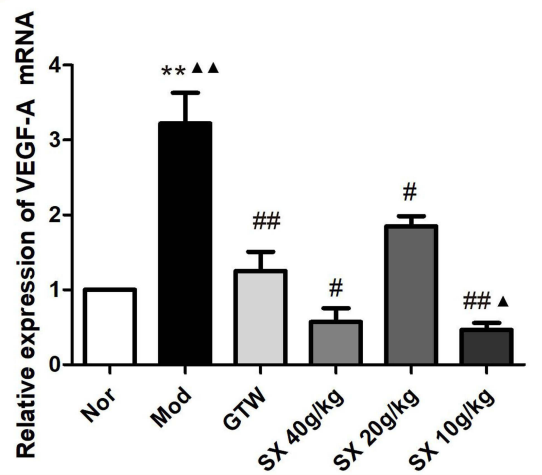

Figure 15 The effect of SX on the mRNA levels of NF-KBp65, STATI, PTGS2, PI3K, AKT and VEGF-A in the rat's joint synovial tissue (Mean \pm SEM, $n=10)$. (A) NF-KBp65:

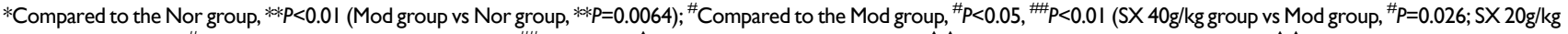

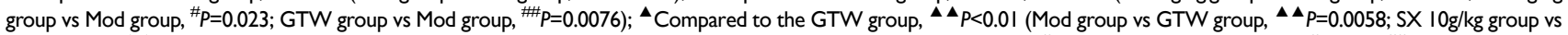

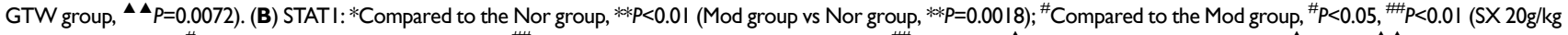

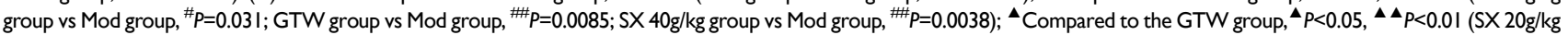

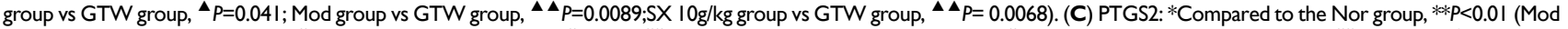

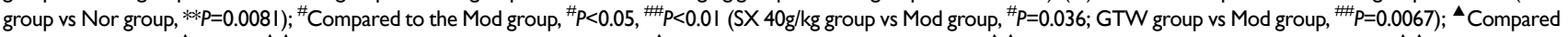

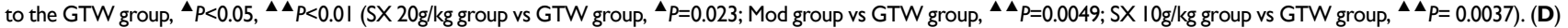
PI3K: *Compared to the Nor group, ${ }^{* * P<0.01 ~(M o d ~ g r o u p ~ v s ~ N o r ~ g r o u p, ~} * * P=0.0074$ ); ${ }^{*}$ Compared to the Mod group, ${ }^{*} P<0.05,{ }^{\#} P<0.01$ (SX 20g/kg group vs Mod group, ${ }^{\#} P=0.024$;

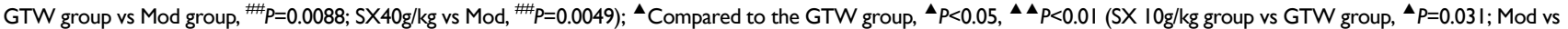
GTW, ${ }^{\wedge} \triangle P=0.0073$ ). (E) AKT: *Compared to the Nor group, ${ }^{* * P} P<0.01$ (Mod group vs Nor group, $* * P=0.0091$ ); ${ }^{*}$ Compared to the Mod group, ${ }^{*} P<0.05$, ${ }^{\#} P<0.01$ (SX 20g/kg group vs

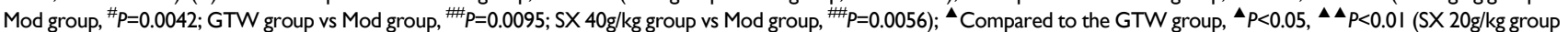
vs GTW group, ${ }^{\boldsymbol{\Delta}} P=0.0431$; Mod group vs GTW group, ${ }^{\boldsymbol{\Delta}} \mathbf{\Delta}=0.0072$; SX 10g/kg group vs GTW group, ${ }^{\boldsymbol{\Delta}} P=0.0075$ ). (F) VEGF-A: *Compared to the Nor group, ${ }^{* *} P<0.01$ (Mod group vs Nor group, $* * P=0.0093$ ); ${ }^{\#}$ Compared to the Mod group, ${ }^{\#} P<0.05$, ${ }^{\# \#} P<0.01$ (SX 40g/kg group vs Mod group, ${ }^{\#} P=0.039 ;$ SX 20g/kg group vs Mod group, ${ }^{\#} P=0.028 ;$ GTW group

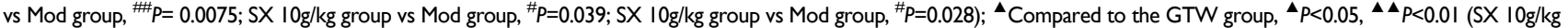
group vs GTW group, ${ }^{\mathbf{A}} p=0.019$; Mod group vs GTW group, $\boldsymbol{\Delta}^{\mathbf{\Delta}} P=0.0035$ ).

Abbreviations: CIA, collagen-induced arthritis; GTW, Tripterygium wilfordii polyglycoside tablet; SX, Sidaxue. 
A

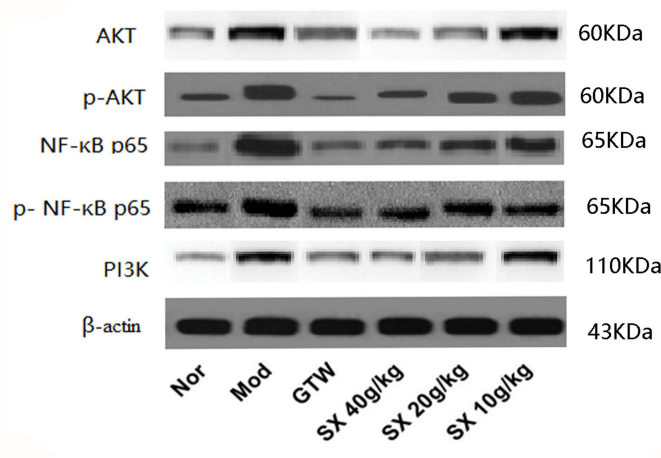

C

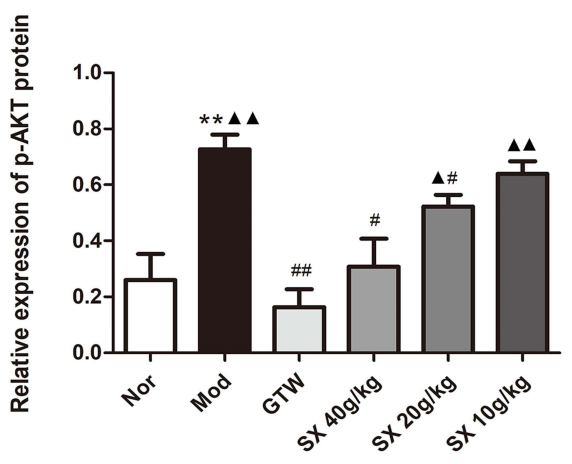

E

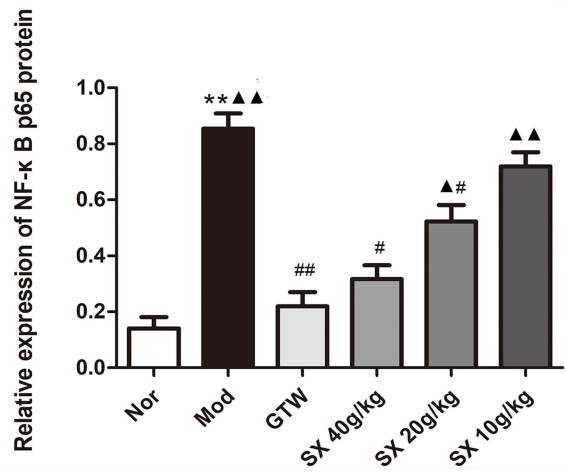

B

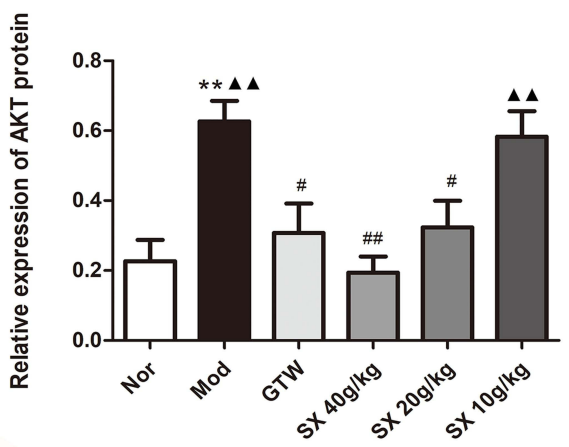

D

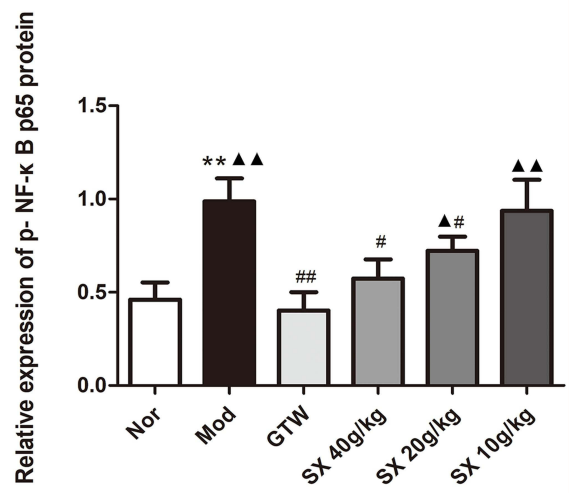

F

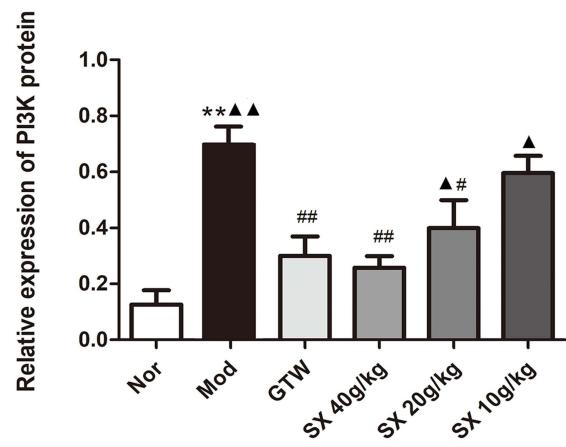

Figure 16 (A) The effect of SX on the protein levels of AKT, p-AKT, NF- $\mathrm{B}$ p $65, \mathrm{p}-\mathrm{NF}-\mathrm{kB}$ p65, PI3K in the rat's joint synovial tissue (Mean \pm SEM, $\mathrm{n}=10$ ). (B) AKT: *Compared to the Nor group, ${ }^{* * P} P 0.01$ (Mod group vs Nor group, ${ }^{* *} P=0.0015$ ); ${ }^{\#}$ Compared to the Mod group, ${ }^{\#} P<0.05,{ }^{\#} P<0.01$ (GTW group vs Mod group, ${ }^{\#} P=0.029 ;$ SX 20 g/kg group vs Mod

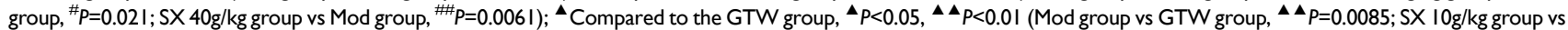
GTW group, $\wedge^{\wedge} P=0.0027$ ). (C) $P$-AKT: $*$ Compared to the Nor group, ${ }^{* * P} P<0.01$ (Mod group vs Nor group, ${ }^{* * P=0.005 I) ; ~}{ }^{\#}$ Compared to the Mod group, ${ }^{\#} P<0.05$, ${ }^{\#} P<0.01$ (SX 40g/

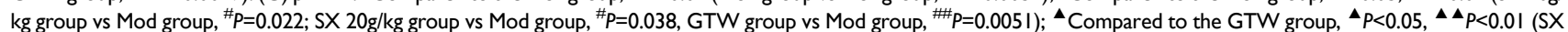

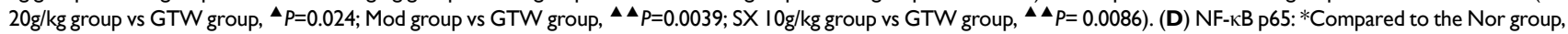
**P<0.0I (Mod group vs Nor group, $\left.{ }^{* *} P=0.007 \mathrm{I}\right)$; ${ }^{\#}$ Compared to the Mod group, ${ }^{\#} P<0.05,{ }^{\#} P<0.01$ (SX $40 \mathrm{~g} / \mathrm{kg}$ group vs Modgroup, ${ }^{\#} P=0.013 ; \mathrm{SX} 20 \mathrm{~g} / \mathrm{kg}$ group vs Mod group, ${ }^{\#} P=$

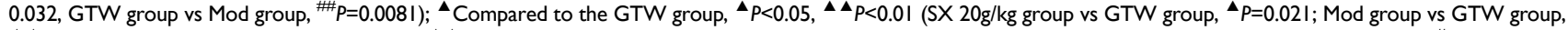

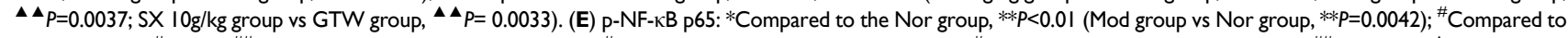
the Mod group, ${ }^{\#} P<0.05,{ }^{\# \#} P<0.01$ (SX 40g/kg group vs Mod group, ${ }^{\#} P=0.042 ; \mathrm{SX} 20 \mathrm{~g} / \mathrm{kg}$ group vs Mod group, ${ }^{\#} P=0.033$, GTW group vs Mod group, ${ }^{\# \#} P=0.0076$ ); ${ }^{\wedge}$ Compared to

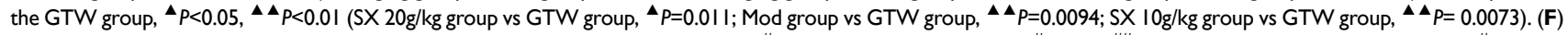
PI3K: *Compared to the Nor group, ${ }^{* *} P<0.01$ (Mod group vs Nor group, ${ }^{* *} P=0.0019$ ); ${ }^{\#}$ Compared to the Mod group, ${ }^{\#} P<0.05$, ${ }^{\#} P<0.01$ (SX 20g/kg group vs Mod group, ${ }^{\#} P=0.039$; GTW group vs Mod group, ${ }^{\#} P=0.0019 ; \mathrm{SX} 40 \mathrm{~g} / \mathrm{kg}$ group vs Mod group, ${ }^{\#} P=0.0062$ ); ${ }^{\wedge}$ Compared to the GTW group, ${ }^{\boldsymbol{\Delta}} P<0.05,{ }^{\Delta}{ }^{\wedge} P<0.01$ (SX 20g/kg group vs GTW group,

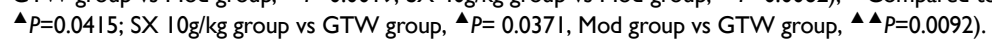

Abbreviations: CIA, collagen-induced arthritis; GTW, Tripterygium wilfordii polyglycoside tablet; SX, Sidaxue. 
AKT phosphorylation level: Compared with the Nor group, the Mod group was significantly up-regulated ( $P=0.0051$, $P<0.01$ ); compared with the Mod group, the SX $40 \mathrm{~g} / \mathrm{kg}$ group, the $\mathrm{SX} 20 \mathrm{~g} / \mathrm{kg}$ group, the GTW group was significantly down-regulated $(P=0.022, P=0.038, P=0.0051, P<0.05)$; There was no statistically significant difference between the SX $10 \mathrm{~g} / \mathrm{kg}$ group and the Mod group $(P>0.05)$, while the difference between the $\mathrm{SX} 40 \mathrm{~g} / \mathrm{kg}$ group, $\mathrm{SX} 20 \mathrm{~g} / \mathrm{kg}$ group and GTW group was not statistically significant $(P>0.05)$ (Figure 16C).

NF-kB p65 total protein expression: Compared with the Nor group, the Mod group was significantly up-regulated ( $P=0.0042, P<0.01$ ); compared with the Mod group, the SX $40 \mathrm{~g} / \mathrm{kg}$ group, SX $20 \mathrm{~g} / \mathrm{kg}$ group, and GTW group were significantly down-regulated $(P=0.042,0.033, P=0.0076, P<0.05, P<0.01)$. There was no significant difference between the SX $10 \mathrm{~g} / \mathrm{kg}$ group and the Mod group $(P>0.05)$, while the difference between the $\mathrm{SX} 40 \mathrm{~g} / \mathrm{kg}$ group and the GTW group was not statistically significant $(P>0.05)$ (Figure 16D).

NF-kB p65 phosphorylation level: Compared with the Nor group, the Mod group was significantly up-regulated ( $P=0.0071, P<0.01$ ); compared with the Mod group, the SX $40 \mathrm{~g} / \mathrm{kg}$ group, the SX $20 \mathrm{~g} / \mathrm{kg}$ group, the GTW group was significantly down-regulated $(P=0.013, P=0.032, P=0.0081, P<0.05, P<0.01)$. There was no significant difference between the SX $10 \mathrm{~g} / \mathrm{kg}$ group and the Mod group $(P>0.05)$, while the difference between the $\mathrm{SX} 40 \mathrm{~g} / \mathrm{kg}$ group, $\mathrm{SX} 20 \mathrm{~g} / \mathrm{kg}$ group and GTW group was not statistically significant $(P>0.05)$ (Figure 16E).

PI3K protein expression: Compared with the Nor group, the Mod group was significantly increased ( $P=0.0019$, $P<0.01$ ); compared with the Mod group, the SX $40 \mathrm{~g} / \mathrm{kg}$ group, SX $20 \mathrm{~g} / \mathrm{kg}$ group, and GTW group were significantly increased $(P=0.0019,0.0062, P=0.039, P<0.01, P<0.05)$. There was no significant difference between the SX $10 \mathrm{~g} / \mathrm{kg}$ group and the Mod group $(P>0.05)$, while the difference between the $S X 40 \mathrm{~g} / \mathrm{kg}$ group and the GTW group was not statistically significant $(P>0.05)$ (Figure 16F).

Through the network pharmacology analysis, the PI3K/AKT signaling pathway and NF-KkB signaling pathway play an important role in the progression of RA disease, and Western Blot results confirmed that SX can reduce AKT, p-AKT, $\mathrm{NF}-\kappa \mathrm{B}$ p65, p-NF-кB p65. The protein content of PI3K may be related to slowing down joint inflammation and pannus, thereby achieving the effect of treating RA.

\section{Discussion}

RA is one of the most prevalent autoimmune disease with no cure. ${ }^{1-3}$ Synovial pannus and synovial inflammation play an important role in RA development. ${ }^{1-3}$ A main feature of RA is the "tumor-like" infiltrating growth of new blood vessels. A large number of inflammatory factors in the inflamed joint cavity are known to induce excessive proliferation of synovial blood vessels and promote the formation of pannus, but the specific mechanism leading to this proliferation is currently unclear. ${ }^{1-3} \mathrm{SX}$ is a classic prescription of Miao medicine for RA treatment used by the ethnic minority Miao people in northern areas of Guizhou Province, China. This study combines network pharmacology and molecular docking to study the molecular mechanism of SX in the treatment of RA and our results provide more evidence that supports further use of SX in RA. ${ }^{13-15}$ Through research, obtained 33 active ingredients of the SX meeting the conditions at the same time, 457 potential targets, 44 targets overlapping with RA, involving 479 GO biological processes and 22 KEGG-related signal pathways (Figures 1-10). It shows that the SX treatment of RA has the characteristics of multiple components, multiple targets, and multiple pathways.

In the network of herbs-active ingredient (Figure 4), the effective compounds were analyzed and arranged in descending order. JXT and FLZXGP play a major role in the compound SX, and the first three active compounds were $\beta$-sitosterol (+)-catechin, ent-Epicatechin. According to the SX-Active Ingredients-RA PPI network, the key ingredients include MOL000358 ( $\beta$-sitosterol), MOL002565 (Medicarpin), MOL000469 (7-oxo- $\beta$-sitosterol), MOL002663 (skimmianine), MOL000392 (formononetin), MOL000497 (licochalcone A). Studies have shown that JXT mainly uses flavonoids in order to play an active role. JXT and FLZXGP can inhibit the proliferation of RA synovial cells, has anti-inflammatory and immunomodulatory effects, and can inhibit the expression of TNF- $\alpha$, IL- $1 \beta$ and other factors in CIA rats to exert its drug activity effect. ${ }^{48}$ FLZX ethanol total ointment and ethyl acetate can effectively inhibit the progression of arthritis in CIA mice. ${ }^{49}$ In addition, studies have shown that DXT and HGT also have therapeutic effects on RA. ${ }^{13-15}$ The important pathological manifestations of RA patients are based on different stages of disease development, joint swelling, heat pain, joint deformity, and even disability may occur. According to the 
arthritis index score and the degree of foot swelling (Figure 12), the SX can improve the pathological manifestations of joint swelling and deformity, and achieve the effect of treating RA. $\beta$-sitosterol and (+)-cajanin have good antiinflammatory and bacteriostatic effects, ${ }^{50}$ among which the anti-inflammatory mechanism may inhibit the activation of inflammasome NLRP3 in epidermal cells and macrophages to inhibit CAS1. The production and activation of the MAPK signaling pathway reduces the expression levels of TNF- $\alpha$, IL-1 $\beta$, IL-6, and IL- 8 in cells, thereby playing an antiinflammatory effect. ${ }^{51}$ In addition, $\beta$-sitosterol has an estrogen-like effect, and can combine with estrogen receptors to exert estrogen or anti-estrogen effects. We note that estrogen is one of the most important substances in regulating the balance of bone metabolism. ${ }^{51}(+)$-Cajanin can inhibit the formation of actin loops in osteoclasts, thereby affecting the maintenance of normal morphology of osteoclasts, ${ }^{52}$ and can also inhibit the expression of genes and proteins related to osteoclast differentiation such as C-fos, Ctsk, Acp5, and hinder the normal differentiation process of osteoclasts. There are many studies on the mechanism of RA bone destruction, of which inflammation-mediated enhancement of osteoclast differentiation is the most important. ${ }^{52,53}$ During RA disease activity, inflammatory mediators such as TNF- $\alpha$, IL-1, IL-6 induce the expression of RANKL, etc., which can increase the secretion of the osteoclast activating factor and reduce the secretion of the osteoblast activating factor, so that the synovial membrane around the joint can be formed. The differentiation of fibroblasts and osteoclasts is accelerated, and the differentiation of osteoblasts is inhibited, leading to the destruction of local articular cartilage and bone and joint dysfunction. ${ }^{52,53}$ In this study (Figure 14B and D), the SX can reduce the expression of TNF- $\alpha$ and IL- 6 , and play a role in the treatment of RA, but the specific mechanism of which active ingredient in SX treats bone destruction needs to be further studied. Medicarpin is a flavonoid component, which has anti-inflammatory, anti-tumor and anti-oxidant characteristics. ${ }^{54}$ Skimmianine is effective in the treatment of rheumatoid arthritis. The histopathological features of RA are: moderate narrowing of the articular cavity, moderate to severe hyperplasia of articular cartilage, severe hyperplasia of synovial tissue and visible pannus formation, while skimmianine can relieve bone and cartilage Erosion, inhibit inflammatory cell infiltration, synovial tissue proliferation, pannus formation and other effects. ${ }^{55,56}$ Licochalcone A has a wide range of pharmacological activities, including antitumor, anti-inflammatory, anti-bacterial, anti-parasitic and so on ${ }^{57,58}$ Formononetin can regulate IL-6, IL-3 and other inflammatory factors to promote the proliferation of hematopoietic cells. ${ }^{59} \mathrm{~A}$ Chinese herbal compound is a complex prescription, and the efficacy of a single component on RA remains to be further studied.

The immune cells that develop RA mainly include synovial B cells, synovial macrophages, neutrophils and T cells. Studies have shown that there are a large number of macrophages in the cartilage tissue and synovial cells of RA patients. ${ }^{60}$ When the synovial macrophages are activated, they can make the histocompatibility complex class II molecules MHC II molecules, chemokines and inflammatory factors. Such overexpression, which in turn leads to synovial tissue hyperplasia, promotes inflammation and aggravates the pathological changes of pannus. Neutrophils account for $60 \%$ of human peripheral blood and are the most important immune cells in the human body. ${ }^{60}$ They have important functions such as sterilization, phagocytosis, and division. Their expression is elevated in the synovial fluid of RA patients. A large number of T cells accumulate in the synovial fluid and synovial tissues of RA. After they bind to MHC II and antigenic polypeptides, they will activate macrophages, etc., and promote the release of inflammatory cytokines such as TNF- $\alpha$ and IL-1, thereby activating the synovial fluid. Membrane fibroblasts and chondrocytes secrete a variety of enzymes that degrade glycoproteins and collagen, thereby destroying tissues and aggravating the condition of RA. ${ }^{61,62}$ In this experiment (Figure 13A-F), the pathological section of the joint synovial tissue showed that after the SX treatments, the infiltration of lymphocytes, monocytes and other inflammatory cells in the synovial tissue and the proliferation of blood vessels and fibrous tissues in the CIA rats Alleviate, and the destruction of cartilage cells is reduced, but the specific mechanism of action needs to be further studied.

According to the SX-Active Ingredients-RA PPI network (Figure 4) and the protein interaction (PPI) network of the SX in the treatment of RA (Figure 3) shows that the key targets include ESR1, CA2, PTPN1, TNF, IL-6, VEGFA, CXCL8, PTGS2, IL-2. Studies have shown that TNF, IL6, PTGS2, IL2, and VEGF-A are highly expressed in RA patients. Among them, TNF, IL6, PTGS2, and IL2 mainly mediate the inflammation of joint synovium, and VEGF-A is an important factor involved in the growth of blood vessels in the body. The pathological manifestations of RA not only show repeated and continuous synovitis, but also show vascular proliferation. The interaction between the two causes the RA condition to become worse. ${ }^{63,64}$ There are a large number of cytokines in the inflammatory joint cavity of RA, 
including IL-1, IL-6, IL-17, IL-2, TNF- $\alpha$, MMPs, etc. When these cytokines are overexpressed, it will promote the RA joint cavity. The internal inflammation continues repeatedly, and inflammation will promote vascular proliferation, promote the expression of VEGF-A, and cause RA synovial pannus. ${ }^{63,64}$ This study shows that SX can reduce the expression of VEGF-A, inhibit inflammation and vascular proliferation, and achieve the effect of treating RA (Figure 14C and F). The repeated action of the two is one of the most important pathogenesis of RA. ${ }^{63,64}$ Prostaglandin endoperoxide synthase 2 (PTGS2), also known as COX-2, is a critical enzyme in prostaglandin biosynthesis and plays an essential role in the inflammatory response. The level of inflammatory factors caused by RA increases can cause overexpression of PTGS2. The overexpression of PTGS2 induces the activation of the NF- $\mathrm{kB}$ signaling pathway and TNF- $\alpha$ signaling pathway, which leads to more severe inflammation in the body. ${ }^{65-67}$ The etiology of RA is complex. In this study (Figures 15 and 16), SX can reduce the expression of TNF- $\alpha$, NF- $\kappa B$ P65, inhibit the expression of PTGS2, and improve RA inflammation, but the intermodulation relationship between TNF- $\alpha$, NF- $\kappa$ B P65 and PTGS2 needs to be further studied. In addition to inflammatory cells and infection factors, it also includes changes in hormone levels, genetic and environmental factors, diet and living habits, and intestinal flora. Studies have shown that changes in hormone levels also have certain incentives. As far as sex hormones are concerned, epidemiological surveys show that male patients with RA have about three times that of women, and the premenopausal incidence of women has increased significantly, surpassing men of the same age. ${ }^{68,69}$ The incidence rate tends to be the same after the age of 80 , so sex hormones are involved in the pathogenesis and development of RA. ${ }^{68,69}$ ESR1 gene polymorphism has a certain relationship with the pathogenesis of knee osteoarthritis. ESR1 gene mutation may increase the risk of knee osteoarthritis, but its exact mechanism still needs further study. ${ }^{68}$

The analysis of GO biological process (Figure 5) and KEGG pathway (Figure 9) shows that GO biological process includes cell proliferation, apoptosis regulation, cytokine regulation, inflammatory response, cell signal transduction regulation7. The key genes (Figures 6-8 and 10) enriched include MMP14, MMP2, MMP12, STAT1, etc. IL2, TNF- $\alpha$, PTGS2, ADORA2A7. Matrix metalloproteinase (MMP) is an important proteolytic enzyme in the organism. Under the stimulation of cytokines, synovial cells and vascular endothelial cells produce a variety of MMPs, breaking the gap between the production and degradation of extracellular matrix. Balance, causing arthritis, is one of the important pathogenesis modes of RA. ${ }^{69}$ Studies have shown that CD147 can promote synovial cells to secrete a variety of matrix metalloproteinases such as: MMP1, MMP3, MMP9 and so on. Vascular endothelial cell growth factor (VEGF) is a multifunctional cytokine that specifically acts on vascular endothelial cells. It is one of the main factors that promote angiogenesis and is currently known to have the strongest effect on blood vessels. ${ }^{70}$ VEGF can bind to many types of receptors, the most important of which is the tyrosine kinase receptor containing 7 globulin-like domains: type I receptor, type II receptor and type III receptor. ${ }^{70,71}$ VEGF binds to these three receptors to induce the migration of vascular endothelial cells and the maintenance of tubular structure. The angiogenesis effect is mainly the result of VEGF activation of VEGFR-2. High expression of VEGF will break the stability of blood vessels, cause basement membrane damage and increase blood vessels. Permeability and inflammation lead to vascular disease, which leads to vascular functional diseases. ${ }^{72,73}$ In RA patients, the high expression of VEGF can make MMP3 and MMP9 in an activated state, and they can then participate in the degradation of extracellular matrix (ECM) and promote the destruction of RA bone. MMP2 can theoretically degrade the important articular cartilage of type I, III, IV, V, VII and XI collagen, fibronectin, laminin and proteoglycan in ECM. It is speculated that MMP2 plays an important role in the degradation of RA cartilage. ${ }^{74}$ However, some studies have found that the levels and activity of MMP2 remain unchanged in RA animal models, and MMP2 knockout mice will develop more severe arthritis, so the mechanism of MMP2 in RA is very complicated. It may have a dual mechanism of protection and disease. The activity of MMP is regulated by the NF- $\mathrm{BB}$ signal transduction pathway. When the body is stimulated by external factors, the NF- $\mathrm{B}$ signal pathway is activated, which leads to the overexpression of downstream MMP, and this then participates in the inflammation response together with other inflammatory factors, and accelerates the joints. One result is destruction of cartilage. ${ }^{75}$ Among them, TNF- $\alpha$ has been shown to play a key role in tissue inflammation, autoimmunity, and is a strong inflammatory cytokine. Targeted biologics agent for TNF- $\alpha$ have been used in the clinic. ${ }^{76}$ In this study, SX can reduce the expression of VEGF-A, TNF- $\alpha$, and NF- $\mathrm{KBp} 65$, but its effect on MMP-related genes needs to be studied in subsequent experiments.The Janus kinase-signal transducer and activator of transcription (JAK/STAT) signaling 
pathway plays a specific and pleiotropic biological function in the process of immune function regulation and other processes, and is an important way of cell signal transduction. A large number of studies have confirmed that the JAK/ STAT pathway is involved in regulating the production and activation of cytokines in the synovium of RA joints, and is involved in the pathophysiological process of the disease. ${ }^{77}$ By activating JAK1, the activation is mainly responsible for RA immune-mediated inflammation. JAK is a non-receptor tyrosine protein kinase in the cytoplasm. The main substrate of JAK is the STAT family. Many cytokines, such as TNF- $\alpha$, IL-1, etc., activate STAT through the JAK/ STAT signal pathway and induce RA inflammation and bone destruction. Studies have shown that STAT1 and STAT6 are expressed in RA synovial tissue, and STAT1 can regulate RA inflammation, bone formation and bone destruction under the action of INF- $\gamma$, IL-6, IL-10 and other cytokines. ${ }^{78}$ The results of this study show that SX can reduce the expression of STAT1 to reduce inflammation and protect bones and joints. Its mechanism of action may be related to SX's ability to down-regulate TNF- $\alpha$ and IL-6. The pathogenesis of RA is complex, and the related molecular mechanisms of action are also diverse ${ }^{79-83}$ (Figure 15). RA synovial vascular proliferation and inflammation are important causes of joint destruction, which are regulated by cytokines, signal pathways and other factors. Among them, VEGF/PI3K/AKT signal and TNF- $\alpha$ signal and IL/NF- $\mathrm{KB}$ signal pathway are closely related to many signal transduction pathways. ${ }^{84,85}$ In recent years, it has been found that there are abundant expressions of angiogenic factors during the evolution of RA synovial lesions. The VEGF/PI3K/AKT signaling pathway exists in a variety of cells and participates in cell proliferation, differentiation, apoptosis, and blood vessel growth process. Phosphatidylinositol 3 kinase (PI3K) is a heterodimer composed of two subunits: a catalytic subunit D (p110) with a molecular weight of $10 \mathrm{kD}$ and a regulatory subunit (P85) with a molecular weight of $85 \mathrm{kD}$. Akt is a downstream signal molecule of PI3K and also has serine/threonine protein kinase activity. The amino acid sequence of its catalytic active center is similar to that of protein kinase A (Proteinkinase A, PKA) and protein kinase $\mathrm{C}$ (Protein kinase $\mathrm{C}, \mathrm{PKC}$ ). The sequence homology is very high, so it is also called PKB. After PI3K is activated, it binds to the intracellular phosphoinositide dependent kinase (PDKl), prompting PDK1 to phosphorylate the Ser308 site of Akt protein to activate Akt. The phosphorylation site in the process of AKT activation is located Ser473 and Thr308 in the active center domain. ${ }^{86,87}$ AKT activation can initiate downstream signaling molecules, the most important of which is Mammalian rapamycin harrowing protein (mTOR), which can continue to transmit downstream signals and produce biological effects such as gene transcription and cell proliferation. ${ }^{84}$ Studies have shown that PI3K/AKT can promote the synthesis of VEGF at the gene level, which is gradually phosphorylated by activating the kinase system, thereby activating the pathway to regulate cytokines, such as VEGF and HIF- $\alpha .{ }^{83}$ In the pathological changes of RA, inflammation of the synovium interacts with pannus, causing the disease to be repeated. The nuclear transcription factor NF- $\kappa B$ signaling pathway mainly includes nuclear factor- $\mathrm{kB}$

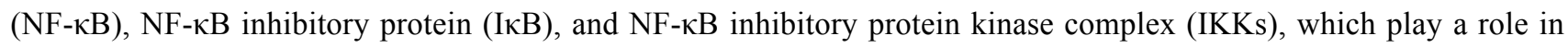
the regulation of inflammation. This is an important role, and is closely related to the occurrence and development of rheumatoid arthritis. ${ }^{88,89} \mathrm{NF}-\mathrm{\kappa B}$ is a protein factor that specifically binds to the enhancer $\mathrm{\kappa B}$ sequence of the immunoglobulin $\kappa$ light chain gene. The dimeric protein composed of P50/P65 is the main component of the active form of NF- $\mathrm{kB}$ in most cells. High levels of TNF- $\alpha$ in the synovial fluid of patients with rheumatoid arthritis can induce the expression of NF-KB ligand activation genes and activate a large number of cytokines, leading to repeated inflammation. ${ }^{88,89}$ The molecular docking results (Figure 11) suggest that $\beta$-amyrin, cajanin, (-)-Catechin gallate, eleutheroside A, Calycosin, $\beta$-sitosterol-3-O- $\beta$-D-glucopyranoside, ormononetin may act on TNF- $\alpha$, VEGF-A, IL-2. Targets such as AKT, PI3K, etc. play a role in the treatment of RA, but the specific role needs to be further studied. Through network pharmacological analysis and animal experiments, it is confirmed that the mechanism of SX treatment of RA may be related to the inhibition of the expression of key factors in the VEGF/PI3K/AKT signaling pathway, TNF- $\alpha$ signaling pathway, and IL/NF- $\kappa B$ signaling pathway.

\section{Conclusion}

In summary, the SX treatment of RA has the characteristics of multiple components, multiple targets, and multiple pathways. SX can improve joint swelling in CIA rats, reduce inflammatory cell infiltration and angiogenesis in joint synovial tissue, and by down-regulating the expression of IL-2, IL-6, TNF- $\alpha$, VEGF, PI3K, AKT, p-AKT, NF-кBp65, p-NF-кBp65, STAT1 and PTGS2are used to control the exacerbation of inflammation and alleviate the proliferation of 
synovial pannus, and at the same time play the role of cartilage protection to achieve the effect of treating RA. The results of this experiment provide a new way to probe deeper into the molecular mechanism of the treatment of RA by the SX and provide new ideas for the study of target therapeutic mechanisms. However, the mechanism of action of specific monomer components and target effects still need to be further studied.

\section{Ethics Statement}

Ethics Approval: The experimental scheme was approved by the Experimental Animal Ethics Committee of Guizhou Medical University (ethical number: 1900868) and followed the guidelines for the ethical review of laboratory animal welfare People's Republic of China National Standard GB/T 35892-2018. The experimental process is strictly in accordance with the National Institutes of Health published guidelines for the care and use of experimental animals (NIH publication 85-23, revised in 1996) and China's guidelines for the ethical review of laboratory animal welfare.

\section{Acknowledgments}

This work was supported by a grant from the National Natural Science Foundation of China (82060776), Science and Technology Program of Guizhou province ([2020]1Y388), and a postgraduate research fund project of the Guizhou Medical University (YJSCXJH [2019]004). We thank Professor Zhenhua Tian of the Guizhou University of Traditional Chinese Medicine for the identification of the four constituent herbs of Sidaxue. Ning Wu and Taohua Yuan contributed equally to this paper and should be considered as the first authors.

\section{Disclosure}

The authors report no conflicts of interest in this work.

\section{References}

1. Madav Y, Barve K, Prabhakar B. Current trends in theranostics for rheumatoid arthritis. Eur J Pharm Sci. 2020;145:105240. doi:10.1016/j. ejps.2020.105240

2. Smolen JS, Aletaha D, Barton A, et al. Rheumatoid arthritis. Nat Rev Dis Primers. 2018;4:18001. doi:10.1038/nrdp.2018.1

3. Kuriya B, Vigod S, Luo J, Widdifield J, Haroon N. The risk of deliberate self-harm following a diagnosis of rheumatoid arthritis or ankylosing spondylitis: a population-based cohort study. PLoS One. 2020;15(2):e0229273. doi:10.1371/journal.pone.0229273

4. Atzeni F, Masala IF, Bagnasco M, Luigi L, Flavio M, Piercarlo S. Comparison of efficacy of ketoprofen and ibuprofen in treating pain in patients with rheumatoid arthritis: a systematic review and meta-analysis. Pain Ther. 2021;10(1):577-588. doi:10.1007/s40122-021-00250-3

5. Zhu CX, Zhou X, Chen HG. Research progress of Miao medicine in the treatment of rheumatoid arthritis. China Mod Appl Pharm. 2020;37 (21):2669-2677.

6. Weyand CM, Goronzy JJ. Immunometabolism in early and late stages of rheumatoid arthritis. Nat Rev Rheumatol. 2017;13(5):291-301. doi:10.1038/nrrheum.2017.49

7. Moltó A, Dougados M. Novel DMARD monotherapy in rheumatoid arthritis. Lancet. 2019;393(10188):2277-2278. doi:10.1016/S0140-6736(19) 30768-8

8. Genovese MC, Kalunian K, Gottenberg JE, et al. Effect of filgotinib vs placebo on clinical response in patients with moderate to severe rheumatoid arthritis refractory to disease-modifying antirheumatic drug therapy: the FINCH 2 randomized clinical trial. JAMA. 2019;322(4):315-325. doi:10.1001/jama.2019.9055

9. Guo YY, Lü SW, Yang BY, et al. HPLC-MS/MS method for the determination and pharmacokinetic study of six compounds against rheumatoid arthritis in rat plasma after oral administration of the extract of Caulophyllum robustum Maxim. J Pharm Biomed Anal. 2020;181:112923. doi:10.1016/j.jpba.2019.112923

10. Chen H, Pan T, Liu P, Wang P, Xu S. Baihu Jia Guizhi decoction improves rheumatoid arthritis inflammation by regulating succinate/SUCNR1 metabolic signaling pathway. Evid Based Complement Alternat Med. 2019;2019:3258572. doi:10.1155/2019/3258572

11. Zhao HR, Zhang Y, Liu B, et al. Identification of characteristic autoantibodies associated with deficiency pattern in traditional chinese medicine of rheumatoid arthritis using protein chips. Front Pharmacol. 2019;10:755. doi:10.3389/fphar.2019.00755

12. Sun K, Szymonifka J, Tian H, Chang Y, Leng JC, Mandl LA. Is use of traditional chinese medicine associated with non-adherence to prescribed western rheumatic medications among Chinese-American patients? A cross-sectional survey. Arthritis Care Res (Hoboken). 2020;72 (10):1474-1480. doi:10.1002/acr.24031

13. Mo XC, Feng GX, Zeng J, Wu N. Anti-inflammatory effects of miao drug sidaxue on rheumatoid arthritis. J Hubei Univ Chin Med. 2015;17 (01):15-18.

14. Wu CX, Wu N, Feng GX, Zeng J. The effects of sidaxue, a kind of Miao National Herb, on TNF- $\alpha$ and IL-1 $\beta$ in adjuvant rheumatoid arthritis rats. J Guiyang Med Coll. 2019;39(01):27-29+34.

15. Wu N, Zeng J, Wang S, Mo X, Tian ZH. Effects of Miao national herbs Sidaxue on rheumatoid arthritis in rats. Chin J Exp Trad Med Formulae. 2014;20(02):120-123.

16. Gao ZJ, Zhu DD, Niu XR, Liu EW, Fu ZF. Review on research progress of chemical constituents and pharmacological activities of Spatholobi Caulis[J/OL]. J Liaoning Univ Trad Chin Med. 2021;41:1-18. 
17. Wang YH, Chen TT, Huang QY. Observation on the efficacy of self made ningleg soup in the treatment of hemodialysis patients with restless legs syndrome. China J Trad Chin Med Technol. 2021;28(05):772-773.

18. Yang NJ, Huo X, Liu WW, Gao YQ, Liu JH. Study on quality standard of Jixueteng granules. Chin Trad Patent Med. $2009 ; 31(01): 144-146$.

19. Zhang Y, Xu X-P, Luo L-Y. Clinical observation on the effect of external divergent shock wave combined with Danggui Jixiteng Decoction in the treatment of inflammatory heel pain of plantar fascia. Zhejiang Univ Trad Chin Med. 2019;14. doi:10.1186/s13020-019-0239-8

20. Wen Z, Chengpeng S, Shuang Z, et al. Recent advances in chemistry and bioactivity of Sargentodoxa cuneata. J Ethnopharmacol. 2021;270:113840. doi:10.1016/j.jep.2021.113840

21. Zhang W, Zhou S, Sun CP, et al. Study on the chemical constituents of Sargentodoxa cuneata. Shanghai J Trad Chin Med. 2020;54(11):85-88.V.

22. Zhu QF, Nie L, Chen Q, Liao SG, Xu GB. Chemical constituents from the roots of Toddalia asiatica(L.) Lam. Cent South Pharm. 2021;19 (09):1800-1805

23. Zeng Z, Tian R, Feng J, Yang NA, Yuan L. A systematic review on traditional medicine Toddalia asiatica (L.) Lam.: chemistry and medicinal potential. Saudi Pharm J. 2021;29(8):781-798. doi:10.1016/j.jsps.2021.05.003

24. Chen L, Tang SQ, Li XJ, et al. A review on traditional usages, chemical constituents and pharmacological activities of periploca forrestii schltr. J Ethnopharmacol. 2021;271:113892. doi:10.1016/j.jep.2021.113892

25. An LL, Liu YC, Liu G, Yang WZ, Jin WY. Research progress on Miao medicine Periploca forrestii Schltr and predictive analysis on quality markers. Chin Arch Trad Chin Med. 2021;39(01):136-141.

26. Zeng DL, Xiong CJ, Wang YG, et al. The shape characteristics and indication scope of the "Sidaxue" of Miao medicine prescription. Chin J Ethnic Med. 2004;58(S1):153-154.

27. Du X, Zhao L, Yang Y, et al. Investigation of the mechanism of action of Porana sinensis Hemsl. against gout arthritis using network pharmacology and experimental validation. Ethnopharmacol. 2020;252:112606. doi:10.1016/j.jep.2020.112606

28. Newman DJ. Modern traditional Chinese medicine: identifying, defining and usage of TCM components. Adv Pharmacol. 2020;87:113-158.

29. Li B, Ma C, Zhao X, et al. YaTCM: yet another traditional Chinese medicine database for drug discovery. Comput Struct Biotechnol J. 2018;16:600-610. doi:10.1016/j.csbj.2018.11.002

30. Yap JJ, Deepak RN, Tian ZZ, et al. The stability of R-spine defines RAF inhibitor resistance: a comprehensive analysis of oncogenic BRAF mutants with in-frame insertion of $\alpha$ C- $\beta 4$ loop. Sci Adv. 2021;7(24):eabg0390. doi:10.1126/sciadv.abg0390

31. Ru J, Li P, Wang J, et al. TCMSP: a database of systems pharmacology for drug discovery from herbal medicines. J Cheminfo. $2014 ; 6: 13$. doi:10.1186/1758-2946-6-13

32. Shanghai Institute of Organic Chemistry, Chinese Academy of Sciences. Chemical professional database[DB/OL]; 1978-2016. Available from: http://www.organchem.csdb.cn. Accessed February 04, 2022.

33. Zhu N, Hou J. Exploring the mechanism of action Xianlingubao prescription in the treatment of osteoporosis by network pharmacology. Comput Biol Chem. 2020;85:107240. doi:10.1016/j.compbiolchem.2020.107240

34. Li S, Zhang B. Traditional Chinese medicine network pharmacology: theory, methodology and application. Chin J Nat Med. 2013;11(2):110-120. doi:10.1016/S1875-5364(13)60037-0

35. Xu Y, Chen WY, Chen ZR, et al. Mechanism of action of Xiaoyao San in treatment of ischemic stroke is related to anti-apoptosis and activation of PI3K/Akt pathway. Drug Des Devel Ther. 2021;15:753-767. doi:10.2147/DDDT.S280217

36. Zhou J, Wang Z, Mao Y, et al. Proteogenomic analysis of pitaya reveals cold stress-related molecular signature. PeerJ. 2020;8:e8540. doi:10.7717/ peerj. 8540

37. Li J, Wang Y, Wang X, Yang Q. CDK1 and CDC20 overexpression in patients with colorectal cancer are associated with poor prognosis: evidence from integrated bioinformatics analysis. World J Surg Oncol. 2020;18:50. doi:10.1186/s12957-020-01817-8

38. Yasmin AZ, Nasser SMI, Gary RM, Nadia MH. A molecular docking study repurposes FDA approved iron oxide nanoparticles to treat and control COVID-19 infection. Eur J Pharm Sci. 2020;153:105465. doi:10.1016/j.ejps.2020.105465

39. Bai Q, Tan S, Xu T, Liu H, Huang J, Yao X. MolAICal: a soft tool for $3 \mathrm{D}$ drug design of protein targets by artificial intelligence and classical algorithm. Brief Bioinform. 2021;22(3):bbaa161. doi:10.1093/bib/bbaa161

40. Jin H, Ma N, Li X, Kang M, Guo M, Song L. Application of GC/MS-based metabonomic profiling in studying the therapeutic effects of Aconitum carmichaeli with Ampelopsis japonica extract on collagen-induced arthritis in rats. Molecules. 2019;24:E1934. doi:10.3390/molecules24101934

41. Peng J, Hu Y, Dong J, Liu Q, Liu Y. Quantifying spatial morphology and connectivity of urban heat islands in a megacity: a radius approach. Sci Total Environ. 2020;714:136792. doi:10.1016/j.scitotenv.2020.136792

42. Liu K, Zhang Y, Liu L, Yuan Q. miR-125 regulates PI3K/Akt/mTOR signaling pathway in rheumatoid arthritis rats via PARP2. Biosci Rep. 2019;39:BSR20180890. doi:10.1042/BSR20180890

43. Mihara T, Tanishima S, Tanida A, Teshima R, Nagashima H. Histological evaluation of lumbar spine changes in rats with collagen-induced arthritis. Yonago Acta Med. 2018;61(1):66-71. doi:10.33160/yam.2018.03.009

44. Chen S, Blijdorp I, van Mens L, et al. IL-17A and IL-17F expression and functional responses in rheumatoid arthritis and peripheral spondyloarthritis. J Rheumatol. 2020;47(11):1606-1613. doi:10.3899/jrheum.190571

45. Diomede F, Marconi GD, Cavalcanti MF, et al. VEGF/VEGF-R/RUNX2 upregulation in human periodontal ligament stem cells seeded on dual acid etched titanium disk. Materials (Basel). 2020;13(3):706. doi:10.3390/ma13030706

46. Fu F, Li LS, Li R, et al. All-trans-retinoid acid induces the differentiation of P19 cells into neurons involved in the PI3K/Akt/GSK3 $\beta$ signaling pathway. J Cell Biochem. 2020;121(11):4386-4396. doi:10.1002/jcb.29659

47. Gong Y, Yu Z, Wang Y, et al. Effect of moxibustion on HIF-1 $\alpha$ and VEGF levels in patients with rheumatoid arthritis. Pain Res Manag. 2019;2019:4705247. doi:10.1155/2019/4705247

48. Zhao YH, Guan D, Jiang M. Foundation and activity of RA. Jiangxi Univ Trad Chin Med. 2019;14. doi:10.1186/s13020-019-0247-8

49. Yang K. The Study on of the analgesic, anti-inflammatory and anti-rheumatoid arthritis activities of extracts form Radix Toddaliae Asiaticae [dissertation]. Huazhong University of Science and Technology; 2012.

50. Qian Y, Delin Y, Yi Z. $\beta$-sitosterol attenuates the intracranial aneurysm growth by suppressing TNF- $\alpha$-mediated mechanism. Pharmacology. 2019;104(5-6):303-311. doi:10.1159/000502221

51. Chen YK, Zeng A, Luo ZH, et al. Progress in the pharmacological action of $\beta$-glusterol. J Guangdong Pharm Univ. $2021 ; 37(01): 148-153$. 
52. Liang ZH. Research of the effect and mechanism of Cajaninstilbene Acid in the prevention and treatment of glucocorticoid-induced ospteoporosis [dissertation]. Guangzhou University of Chinese Medicine; 2020.

53. Zhao XQ, Wei G, Wang Y. Wang Yue experience in rheumatoid arthritis. Shandong J Trad Chin Med. 2021;40(02):175-177+206.

54. Mohd NM, Ashutosh R, Priyanka S, et al. Medicarpin prevents arthritis in post-menopausal conditions by arresting the expansion of TH17 cells and pro-inflammatory cytokines. Int Immunopharmacol. 2020;82:106299. doi:10.1016/j.intimp.2020.106299

55. Eshonov MA, Vinogradova VI, Rasulova KA, Turgunov KK, Tashkhodzhaev B. Ipso-substitution in derivatives of the quinoline alkaloid skimmianine. Chem Nat Compounds. 2021;57:507-511.

56. Zheng YL. Research progress on chemical constituents and pharmacological effects of Yinyu. J Gansu Univ Trad Chin Med. 2018;35(04):93-95.

57. Liu XH, Xing Y, Li MY, et al. Licochalcone A inhibits proliferation and promotes apoptosis of colon cancer cell by targeting programmed cell death-ligand 1 via the NF-кB and Ras/Raf/MEK pathways. J Ethnopharmacol. 2021;273:113989. doi:10.1016/j.jep.2021.113989

58. Zhang HY, Huang YS, Wang H, Wei XN. The effect of licorice chalcone A on osteoarthritis in rats and its relationship with p38-MAPK inflammatory signaling pathway. Chin Prim Med. 2021;35(04):93-95. doi:10.1007/s00052-021-00013-5

59. Sugimoto M, Ko R, Goshima H, Koike A, Shibano M, Fujimori K. Formononetin attenuates H2O2-induced cell death through decreasing ROS level by PI3K/Akt-Nrf2-activated antioxidant gene expression and suppressing MAPK-regulated apoptosis in neuronal SH-SY5Y cells. NeuroToxicology. 2021;85:186-200. doi:10.1016/j.neuro.2021.05.014

60. Xie XQ, Wang YL, Luo SS, Zhao ZY, Li XT. Pathogenesis of rheumatoid arthritis. Digest Worlds Latest Med Info. $2019 ; 28(05): 738-743$.

61. Navrátilová A, Bečvář V, Baloun J, et al. S100A11 (calgizzarin) is released via NETosis in rheumatoid arthritis (RA) and stimulates IL-6 and TNF secretion by neutrophils. Sci Rep. 2021;11(1):6063. doi:10.1038/s41598-021-85561-3

62. Jin SW, Sun SY, Ling HZ, et al. Protectin DX restores Treg/T17 cell balance in rheumatoid arthritis by inhibiting NLRP3 inflammasome via miR-20a. Cell Death Dis. 2021;12(3):280. doi:10.1038/s41419-021-03562-6

63. Wehmeyer C, Frank S, Beckmann D. Sclerostin inhibition promotes TNF-dependent inflammatory joint destruction. Sci Transl Med. 2016;8 (330):330ra35. doi:10.1126/scitranslmed.aac4351

64. Nakamura H, Shimamura S, Ectopic YS. RASGRP2 (CalDAG-GEFI) expression in rheumatoid synovium contributes to the development of destructive arthritis. Ann Rheum Dis. 2018;77(12):1765-1772. doi:10.1136/annrheumdis-2018-213588

65. Petrovská N, Prajzlerová K, Vencovský J, Šenolt L, Filková M. The pre-clinical phase of rheumatoid arthritis: from risk factors to prevention of arthritis. Autoimmun Rev. 2021;20(5):102797. doi:10.1016/j.autrev.2021.102797

66. Meserve J, Luo J, Zhu W, et al. Paternal exposure to immunosuppressive and/or biologic agents and birth outcomes in patients with immune-mediated inflammatory diseases. Gastroenterology. 2021;161(1):107-115. doi:10.1053/j.gastro.2021.03.020

67. Xiao SJ, Zhou YF, Wu Q, et al. FCER1G and PTGS2 serve as potential diagnostic biomarkers of acute myocardial infarction based on integrated bioinformatics analyses. DNA Cell Biol. 2021;40:1064-1075. doi:10.1089/dna.2020.6447

68. Bao L, Zhang B, Zhao C, Liu ZP, Zhou T. Study on the correlation between ESR1 gene polymorphism and the risk of knee osteoarthritis. $J$ Pract Orthoped. 2020;26(10):888-891.

69. Wang HB, Yu WR. Progress in MMP-2/MMP-9 studies in inflammation. Med Rev. 2014;20(17):3120-3122.

70. Chen L, Li JS, Ke X, et al. Chemical profiling and the potential active constituents responsible for wound healing in Periploca forrestii Schltr. J Ethnopharmacol. 2018;224:230-241. doi:10.1016/j.jep.2018.04.023

71. Jia ZR, Wang XT, Wang XY, et al. Calycosin alleviates allergic contact dermatitis by repairing epithelial tight junctions via down-regulating HIF1a. J Cell Mol Med. 2018;22(9):4507-4521. doi:10.1111/jcmm.13763

72. Akagi T, Mukai T, Mito T, et al. Effect of angiotensin II on bone erosion and systemic bone loss in mice with tumor necrosis factor-mediated arthritis. Int J Mol Sci. 2021;21(11):4145. doi:10.3390/ijms21114145

73. Braz NFT, Pinto MRC, Vieira ÉLM, et al. Renin-angiotensin system molecules are associated with subclinical atherosclerosis and disease activity in rheumatoid arthritis. Mod Rheumatol. 2021;31(1):119-126. doi:10.1080/14397595.2020.1740418

74. An S, Bai RC, Li GG, Zhu L, Yang YQ. Effects of Fulingwan modified and subtracted recipe on MMP-3, MMP-13 and TIMP-1 expression in rabbit knee osteoarthritis model. Shanghai J Trad Chin Med. 2021;55(07):64-67.

75. Muniz-Bongers LR, McClain C, Saxena M, Bongers G, Merad M, Bhardwaj N. MMP2 and TLRs modulate immune responses in the tumor microenvironment. JCI Insight. 2021;6(12):e144913. doi:10.1172/jci.insight.144913

76. Li Y, Qi WF, Shi YQ, Wang MM. Clinical effect of TNF- $\alpha$ antagonist in the treatment of rheumatoid arthritis. Chin J Nosocom Infect. 2021;31 (01):73-77.

77. Yang S, Jiang M, Wei X. Based on JAK/STAT pathway to explore the anti-inflammatory effect of Guizhi Shaoyao Zhimu Decoction on MH7A cells induced by TNF- $\alpha$. Chengdu Univ Trad Chin Med. 2019;14. doi:10.1186/s13020-019-0227-z

78. Leclair NK, Knopf J, Baldwin M, Forouhar F, Onyiuke H. Rheumatoid pannus presenting as a large epidural mass in the subaxial cervical spine: a case report. Neurochirurgie. 2021;68:SS0028-5. doi:10.1016/j.neuchi.2021.02.009

79. Shang ZZ, Qin DY, Li QM, et al. Dendrobium huoshanense stem polysaccharide ameliorates rheumatoid arthritis in mice via inhibition of inflammatory signaling pathways. Carbohydr Polym. 2021;258:117657. doi:10.1016/j.carbpol.2021.117657

80. Gan DH, Cheng WX, Ke LQ, et al. Repurposing of pirfenidone (anti-pulmonary fibrosis drug) for treatment of rheumatoid arthritis. Front Pharmacol. 2021;12:631891. doi:10.3389/fphar.2021.631891

81. Wu G, Nie W, Wang Q, et al. Umbelliferone ameliorates complete freund adjuvant-induced arthritis via reduction of NF- $\kappa \mathrm{B}$ signaling pathway in osteoclast differentiation. Inflammation. 2021;44(4):1315-1329. doi:10.1007/s10753-021-01418-X

82. Guo X, Ji J, Jose Kumar Sreena GS, et al. Panax japonicusComputational prediction of antiangiogenesis synergistic mechanisms of total saponins of against rheumatoid arthritis. Front Pharmacol. 2021;11:566129. doi:10.3389/fphar.2020.566129

83. Wang L, Pu X, Nie X, et al. Integrated serum pharmacochemistry and network pharmacological analysis used to explore possible anti-rheumatoid arthritis mechanisms of the Shentong-Zhuyu decoction. J Ethnopharmacol. 2021;273:113988. doi:10.1016/j.jep.2021.113988

84. Damian L, Lebovici A, Pamfil C, Belizna C, Vulturar R. Rheumatoid arthritis and CLOVES syndrome: a tricky diagnosis. Diagnostics (Basel). 2020;10(7):467. doi:10.3390/diagnostics10070467

85. Liu C, He L, Wang J, et al. Anti-angiogenic effect of Shikonin in rheumatoid arthritis by downregulating PI3K/AKT and MAPKs signaling pathways. J Ethnopharmacol. 2020;260:113039. doi:10.1016/j.jep.2020.113039 
86. Jacinto E, Facchinetti V, Liu D, et al. SIN1/MIP1 maintains rictor-mTOR complex integrity and regulates Akt phosphorylation and substrate specificity. Cell. 2006;127(1):125-137. doi:10.1016/j.cell.2006.08.033

87. Yang J, Cheng M, Gu B, Wang J, Yan S, Xu D. CircRNA_09505 aggravates inflammation and joint damage in collagen-induced arthritis mice via miR-6089/AKT1/NF-kB axis. Cell Death Dis. 2020;11(10):833. doi:10.1038/s41419-020-03038-z

88. Loh C, Park SH, Lee A, Yuan R, Ivashkiv LB, Kalliolias GD. TNF-induced inflammatory genes escape repression in fibroblast-like synoviocytes: transcriptomic and epigenomic analysis. Ann Rheum Dis. 2019;78(9):1205-1214. doi:10.1136/annrheumdis-2018-214783

89. Zhou HF, Yan HM, Pan H, et al. Peptide-siRNA nanocomplexes targeting NF-kB subunit p65 suppress nascent experimental arthritis. J Clin Invest. 2014;124(10):4363-4374. doi:10.1172/JCI75673

Drug Design, Development and Therapy

\section{Publish your work in this journal}

Drug Design, Development and Therapy is an international, peer-reviewed open-access journal that spans the spectrum of drug design and development through to clinical applications. Clinical outcomes, patient safety, and programs for the development and effective, safe, and sustained use of medicines are a feature of the journal, which has also been accepted for indexing on PubMed Central. The manuscript management system is completely online and includes a very quick and fair peer-review system, which is all easy to use. Visit http://www.dovepress.com/testimonials.php to read real quotes from published authors.

Submit your manuscript here: https://www.dovepress.com/drug-design-development-and-therapy-journal 